\title{
Autonomous Discovery of Motor Constraints in an Intrinsically Motivated Vocal Learner
}

\author{
Juan Manuel Acevedo-Valle, Cecilio Angulo, and Clement Moulin-Frier
}

\begin{abstract}
This paper introduces new results on the modeling of 2 early vocal development using artificial intelligent cognitive archi3 tectures and a simulated vocal tract. The problem is addressed 4 using intrinsically motivated learning algorithms for autonomous 5 sensorimotor exploration, a kind of algorithm belonging to the 6 active learning architectures family. The artificial agent is able 7 to autonomously select goals to explore its own sensorimotor \& system in regions, where its competence to execute intended goals is improved. We propose to include a somatosensory system to provide a proprioceptive feedback signal to reinforce learn11 ing through the autonomous discovery of motor constraints. ${ }_{12}$ Constraints are represented by a somatosensory model which 13 is unknown beforehand to the learner. Both the sensorimotor 14 and somatosensory system are modeled using Gaussian mixture 15 models. We argue that using an architecture which includes a 16 somatosensory model would reduce redundancy in the sensori17 motor model and drive the learning process more efficiently than 18 algorithms taking into account only auditory feedback. The role 19 of this proposed system is to predict whether an undesired colli20 sion within the vocal tract under a certain motor configuration 21 is likely to occur. Thus, compromised motor configurations are 22 rejected, guaranteeing that the agent is less prone to violate its 23 own constraints.
\end{abstract}

Index Terms-Active learning, early vocal development, 25 Gaussian mixture models (GMMs), intrinsic motivations, sen26 Sorimotor exploration.

27

28

${ }_{20} \mathbf{1}$ $\mathrm{N}$ RECENT years, there has been an increasing interest in using robots to perform daily life activities in the so presence of humans. As robot-human interactions become ${ }_{31}$ common then human-like communication systems become 32 more relevant to robotics. Speech is one of the most studied зз communication systems because it allows human-spoken lan34 guage. However, as mentioned in [1], the idea that speech is a 35 deeply encrypted "code" prevails among the speech specialists

Manuscript received July 19, 2016; revised November 18, 2016 and February 20, 2017; accepted April 14, 2017. This work was supported by the PATRICIA Research Project through the Spanish Ministry of Economy and Competitiveness under Grant TIN2012-38416-C03-01. The work of J. M. Acevedo-Valle was supported by CONACYT under Grant 216872 . (Corresponding author: Juan Manuel Acevedo-Valle.)

J. M. Acevedo-Valle and C. Angulo are with the GREC Research Group, Universitat Politècnica de Catalunya, 08028 Barcelona, Spain (e-mail: juan.manuel.acevedo.valle@upc.edu).

C. Moulin-Frier was with Flowers team, Inria/ENSTA-Paristech, 33405 Bordeaux, France. He is now with the SPECS Laboratory, Universitat Pompeu Fabra, 08018 Barcelona, Spain.

Color versions of one or more of the figures in this paper are available online at http://ieeexplore.ieee.org.

Digital Object Identifier 10.1109/TCDS.2017.2699578 and cracking this code is still an unsolved problem. Some of 36 the mysteries about speech might be solved if we are able to understand all the mechanisms underlying early speech acquisition in children. Thus, this paper, provides new results to contribute to the study of early speech development using machines.

Developmental robotics is a relatively novel approach, it 42 aims at understanding and modeling the role of developmental ${ }_{43}$ processes in the emergence of complex behaviors, including 44 social ones. Its goal is twofold, on the one hand it is used to 45 build more efficient cognitive machines applying developmen- 46 tal theories, and on the other hand it also provides insights into $\quad 47$ human developmental mechanisms, especially during infancy. 48 A deeper understanding of these mechanisms would explain 49 how human beings develop from infancy to functional adults 50 capable of solving highly complex cognitive tasks [2].

Autonomous robot design could notably benefit from 52 the available knowledge of biological science and self- 53 organization theories [3]. Deep understanding of the embod- 54 iment paradigm is paramount to integrate that knowledge 55 into robotics. This paradigm is also well represented by 56 the quote "understanding by building" [4]. It states that the ${ }_{57}$ behavior of an agent is not only the result of a system 58 control structure, but also a result of complex interactions 59 with its ecological niche, its morphology, and its material 6 properties [3], [4].

In this paper, language emergence is studied according 62 to behavioral and neurophysiological evidence, moreover the role of motor constraints is especially considered. The main assumption is that early vocal development can be studied as a result of embodiment, self-organization, and emergence 66 mechanisms produced by human evolution. In general, studies ${ }_{67}$ have shown that infants show preparedness to acquire natural 68 language. Motor, perceptual, social, and learning ability con- 69 straints, and their maturation during infant development play 70 a key role in the emergence of language [1].

Equally important, machine learning techniques have 72 rapidly evolved, providing developmental robotics with 73 interesting approaches as active learning. In contrast to the 74 more usual passive learning algorithms, active learning data 75 are collected in order to minimize a given property of the 76 learning process, e.g., the uncertainty [5] or the prediction 77 error [6] of a model. This family of algorithms is of partic- 78 ular interest for developmental robotics. During sensorimotor 79 exploration they allow the agent to focus on parts of the sen- 80 sorimotor space in which exploration is expected to improve 81 the quality of the learned model [7]. 
83 The contribution of this paper is extending the study 84 of early language development using intrinsically motivated 85 exploration algorithms. Herein, we provide new simulation 86 results showing the suitability of these algorithms in the self87 exploration of sensorimotor vocal spaces. The theoretical basis 88 of the probabilistic models used to represent knowledge is also 89 provided. Furthermore, we propose an architecture that could 90 be used to study the role of constraints during sensorimotor 91 exploration in embodied agents. Finally, it is worth mentioning 92 that the learning algorithm presented herein could be applied 93 to any system subjected to constraints in order to improve 94 learning progress.

95 The remainder of this paper is organized as follows. 96 Section II introduces related works. Section III highlights 97 the role of intrinsic motivations and proprioceptive feedback 98 in vocal development. The experiment setup is described 99 in Section IV and results are presented and discussed in 100 Section V. Finally, the conclusions are presented in Section VI.

\section{1 \\ II. RELATED WORK}

102 This paper revisits and expands the investigation introduced 103 in [8] and [9]. In [9], an intrinsically motivated exploration 104 architecture was proposed for the study of the developmental 105 stages emergent during the early vocal development of infants. 106 For the experimentation the simulated ear-vocal tract model 107 DIVA [10] was used. In spite of the relevance of its results, 108 the motor constraints and the somatosensory system were 109 neglected in [9]. However, morphological constraints play a 110 key role in speech acquisition. Therefore, a new exploration 111 algorithm proposed in [8] to incorporate motor constraints 112 awareness using a somatosensory model. In the past, some 113 studies have tried to explain the emergence of developmental 114 stages during the vocal development, assuming their exis115 tence, but those stages were bridged using hard-coding for 116 experimentation [10]-[13].

117 In [14], an approach for inverse kinematics learning in 118 redundant systems was presented. It was demonstrated that 119 goal babbling can be advantageous in learning in the early 120 stages of development, as observed in developmental theo121 ries. In parallel, [15] presented an intrinsically motivated goal 122 exploration approach for the active learning of inverse mod123 els. This approach was applied to the vocal sensorimotor space 124 exploration in [9] and [16]. The algorithm considered in this 125 paper extends intrinsically motivated exploration in the goal 126 space to include motor constraints. Considering both motor 127 and perceptual constraints during learning and exploration is 128 crucial to design cognitive architectures for motor control.

129 Among the efforts to model the acquisition of speech there 130 is the DIVA model [10]. It aims to imitate the underlying 131 neurophysiological mechanisms for speech acquisition and 132 production. The cognitive architecture of the system is an ${ }_{133}$ artificial neural network. The model includes the premotor, 134 motor, auditory and somatosensory cortical areas, and simu135 lated ear-vocal tract system. In [10], the somatosensory model 136 was effectively integrated into the acquisition and production 137 of speech processes. It was not used as an element to integrate 138 motor constraints but as an extra source of sensory-feedback.
The ear-vocal tract component of the DIVA model is used in 139 this paper, as it was in [8] and [9].

Finally, another interesting contribution was the active learn- 141 ing architecture presented in [17] which considered time con- 142 straints. This paper proposed a music performance imitation 143 scenario and implemented a learning architecture able to learn 144 a musical instrument model and a body capabilities model; the 145 architecture is also able to imitate a sequence of sound, while ${ }_{146}$ simultaneously kinematic errors, due to the control architec- 147 ture, are corrected. Similar to [9], models employed in [17] 148 were based on Gaussian mixture models (GMMs).

\section{Early Vocal Development in Machines}

Human speech production is one of the most complex motor 151 acts performed by any living being [18]. Producing a linguis- 152 tic message that can be understood by another human requires 153 coordinating many degrees of freedom in the respiratory, 154 laryngeal, and supraglottal articulatory system.

155

How infants acquire the complex ability to control speech 156 production and in general how they learn language remains a 157 matter of research [1]. It has been pointed out that strong 158 regularities can be observed in the structure of the vocal 159 development process independently of interindividual differ- 160 ences [1], [19]. In general, the infant first discovers how to 161 control phonation, then focuses on vocal variations of unartic- 162 ulated sounds and finally automatically discovers and focuses 163 on babbling with articulated proto-syllables. In [18], some 164 experiments suggested that goals of speech movements are 165 auditory in nature and maintenance of motor command maps 166 to auditory results is performed with auditory feedback. $\quad{ }_{167}$

It is important to inquire into the developmental assump- 168 tions considered in the experiments in [8] and [9], as this 169 paper is based on those experiments. Regarding the infant 170 development stages mentioned in [1], our experiments con- 171 sider the developmental stage known as canonical babbling 172 (CB) [20] and the beginning of language-specific speech pro- 173 duction [1]. Results suggest that during CB infants learn to 174 control their ear-vocal tract system based on auditory feed- 175 back. Nevertheless, when infants begin to babble they do it 176 regardless of the audibility of their vocalizations. CB could 177 be the result of a natural tendency of infants to move their 178 body parts rhythmically motivated by sensory feedback [20]. 179

Consistent with the theory, we assumed a simplified expla- 180 nation that the artificial agent is exploring its ear-vocal tract 181 system choosing auditory goals and evaluating the result. 182 Therefore, our cognitive architecture allows the agent to 183 explore regions, where the competence to produce intended 184 sounds is improved. However, we also endow the agent with 185 autonomous mechanisms to discover constraints in order to 186 drive the exploration. To accomplish that objective, previously ${ }_{187}$ proposed active learning architectures and the proprioceptive 188 feedback concept are combined.

\section{A. Intrinsically Motivated Exploration Architectures}

Among the vast number of active learning architectures, 191 this paper considers the exploration architecture proposed 192 
193 by [15]. This architecture reproduces the formalism of intrin194 sic motivation inspired by psychological literature as proposed 195 previously in [21] and [22]. Using goal babbling, intrinsi196 cally motivated exploration aims to minimize the error of an 197 agent to reach self-generated goals measured according to a 198 competence function. This architecture allows artificial agents 199 to efficiently and actively explore and generate maps from 200 motor capacities to perceived results. Therefore, exploration 201 occurs over regions in which agents perceive they are becom202 ing more competent to reach self-generated goals. Intrinsically 203 motivated exploration architectures were originally designed 204 to actively learn inverse models of high-dimensional input205 output spaces. This architecture was later extended by [9] to 206 study self-organization in early vocal development stages in 207 infants and robots.

208 Intrinsically motivated learning algorithms have shown 209 favorable results in previous experiments to learn sensorimotor 210 coordination skills in redundant nonlinear high-dimensional 211 mappings which share many mathematical properties with 212 vocal spaces. Moulin-Frier et al. [9] used a simulated ear213 vocal tract system to study the emergence of developmental 214 stages implementing intrinsically motivated exploration. They 215 argued that the development of the agent self-organizes into 216 vocal developmental sequences. The results presented therein 217 opened the door to a new approach in vocal development 218 to be explored. This paper introduces a methodology which 219 enhances intrinsically motivated architectures with constraint 220 awareness.

\section{B. Proprioceptive Feedback}

222 Some of the most adopted theories of speech state that 223 speech production is organized in terms of motor control sig224 nals and their associated vocal tract configurations which has 225 been corroborated by several experimental results [23], [24]. 226 Nevertheless, we adopt the simplified hypothesis that speech 227 goals are defined acoustically and maintained by auditory 228 feedback [18]. CB is a rhythmic behavior that, with some dif229 ferences, emerges in both, normally developing infants and 230 infants with hearing loss. When infants start to babble, they ${ }_{231}$ do it regardless of the audibility result (i.e., they produce audi232 ble and voiceless vocalizations). However, evidence suggests 233 that, around the onset of $\mathrm{CB}$, infants learn to vocalize based 234 on auditory feedback [1], [20].

${ }_{235}$ How the somatosensory system ${ }^{1}$ affects the ear-vocal tract 236 exploration is an open question that was not previously ${ }_{237}$ approached in [9]. However, the relevance of the somatosen238 sory system for speech has been shown in different exper239 iments, for instance the results in deaf individuals suggest 240 that somatosensory inputs related to movement play a more 241 important role in speech production than what was thought 242 before [25], [26]. Furthermore, the fact that CB also emerges 243 in deaf infants suggests that somatosensory feedback must play 244 a more relevant role during the prelinguistic vocal development 245 in infants [27].

\footnotetext{
${ }^{1}$ Strictly, the somatosensory system is also a sensorimotor system. In future works, we will distinguish two sensorimotor systems: 1) the auditory-motor system and 2) the somatosensory-motor system.
}

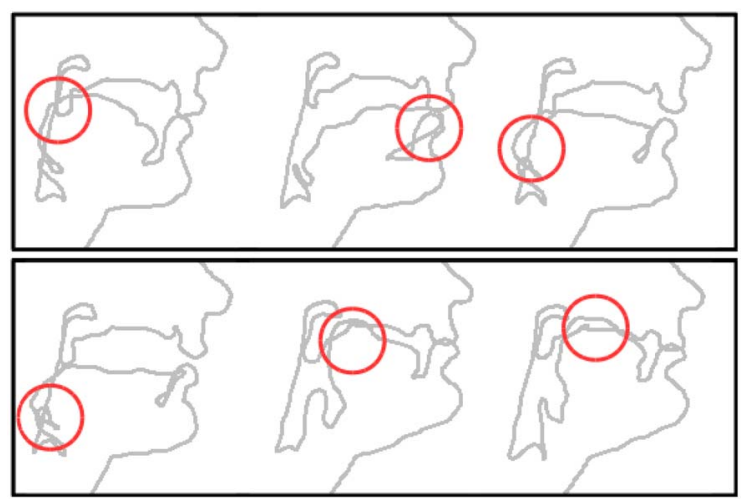

Fig. 1. Examples of articulatory configurations that produce collisions in the DIVA vocal tract model.

In [28], a robotic device able to generate patterns of facial 246 skin deformation related to certain speech productions was 247 used. The results showed that when the facial skin is stretched 248 whilst subjects are listening to words, the sounds they hear are 249 altered. Thus, theory and results suggest that the somatosen- 250 sory system is involved in speech perception. Following 251 this hypothesis, improvements can made to the experiments 252 proposed in [9] by including a somatosensory system to endow 253 the learner with physical constraint awareness. 254

In [8], the foundations of a simplified architecture were 255 established allowing us to include physical constraints to the 256 learning process through a proprioceptive signal, similar to ${ }_{257}$ the ability to feel pain in humans. The open source DIVA ${ }_{258}$ model $^{2}[10]$ provides a synthesizer that represents the human 259 vocal tract and ear systems. The DIVA model also includes 260 a somatosensory system, but in spite of it, there is a lack of 261 physical constraints in the DIVA vocal tract. The absence of 262 constraints allows the execution of motor commands that lead 263 to collisions or articulatory superpositions. Both circumstances 264 lead to no phonation and moreover, the latter is a contradictory 265 result since it lacks physical sense, as shown in Fig. 1.266

To overcome the drawbacks caused by the lack of con- 267 straints, we introduce a somatosensory system. This new 268 element, not considered in [9], is based on an area function 269 which is a vector descriptor of the vocal tract shape. It con- 270 sists of a mechanism that evaluates if an exploratory motor 271 command produces a collision or superposition of articulatory 272 tissues, the system generates a proprioceptive signal. Using the 273 data generated with this mechanism, the agent builds a map 274 from motor commands to proprioceptive results. This map is 275 used to predict which motor commands may lead to undesired 276 collisions, so they may be rejected, forcing the agent to choose 277 a new auditory goal. In the next section, this mechanism is 278 explained in detail.

\section{Proposed Architecture}

280

The experimental architecture proposed in this paper to 281 study the early vocal development in machines is shown 282 in Fig. 2, where five elements interact. These elements are 283

\footnotetext{
${ }^{2} \mathrm{http}: / /$ www.bu.edu/speechlab/software/diva-source-code/
} 


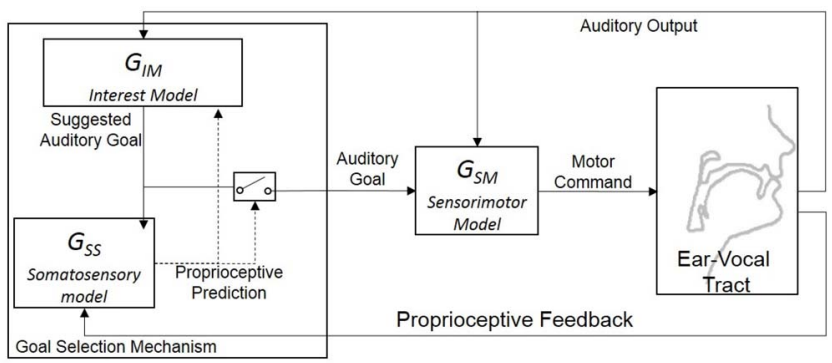

Fig. 2. Experimental architecture. It is composed by five interacting modules, two of them contained within the ear-vocal tract module (the sensorimotor system and the somatosensory system).

284 introduced below and explained in detail in the coming 285 sections.

\section{A. Sensorimotor System}

${ }_{310}$ The DIVA vocal tract configuration is determined by the 311 position of ten articulators and three phonation parameters. ${ }_{312}$ Along this paper, only seven articulators and two phonation 313 parameters (voicing and glottal pressure) are considered [9]. 314 Articulators and voicing parameter motor dynamics are mod315 eled as overdamped second order systems

$$
\ddot{x}+2 \zeta \omega_{0} \dot{x}+\omega_{0}^{2}(x-m)=0
$$

317 with $\zeta=1.01$ and $\omega=(2 \pi / 0.8)$ representing the damping 318 factor and the natural frequency, respectively. The duration of 319 each vocal experiment in seconds is 0.8 , whereas $m$ and $x$ rep320 resents the desired articulator position (motor command) and 321 the current articulator position, respectively. During each vocal 322 experiment two different motor commands are introduced for 323 each of the seven articulators and the two voicing parameters: 324 one for the $0-250 \mathrm{~ms}$ window and another for the remain325 ing time. Thus, each motor command is represented by an

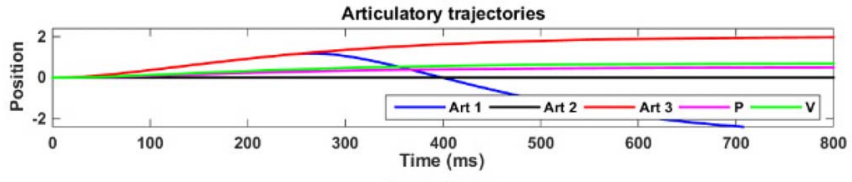

Sound wave
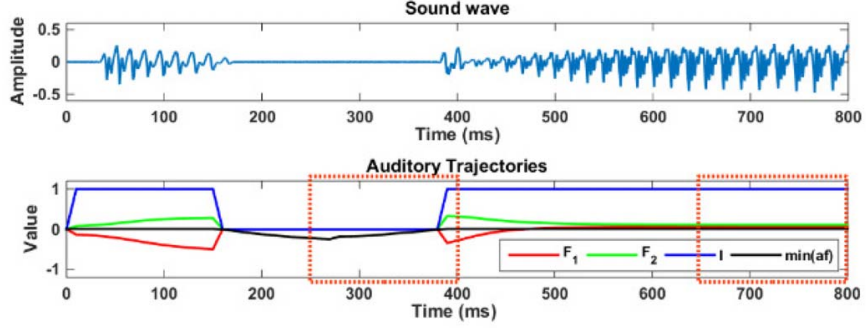

Fig. 3. Vocalization experiment structure. The upper plot shows the articulatory trajectories, from 0 to $250 \mathrm{~ms}$, the commands for Art1, Art2, and Art3 are set to 2, 0 , and 2, respectively, whereas the glottal pressure and voicing are both set to 0.5 . From 250 to $800 \mathrm{~ms}$, the commands for Art1, Art2, and Art3 are set to $-3,0$, and 2, respectively, whereas the glottal pressure and voicing are both set to 0.7 . The remaining motor commands are set to zero. The middle plot represents the speech sound wave signal. The bottom plot shows the auditory trajectories. The dotted outlined boxes represent the perception time windows from 250 to $400 \mathrm{~ms}$ and the second from 650 to $800 \mathrm{~ms}$. The auditory output $s$ are determined from the average of each trajectories along each one of the time windows. Whereas the proprioceptive feedback $p$ is determined by the average value of $\min \left(a_{f}\right)$.

18-D vector. The auditory output of a human vocalization can 326 be described by its formant frequencies. We consider the first ${ }_{327}$ two formant frequencies, $F_{1}$ and $F_{2}$, along with an intonation 328 signal $I$. The intonation signal is 1 when phonation occurs ${ }_{329}$ and 0 otherwise, two conditions are required for phonation 330 to occur: 1) the area function $a_{f}$ of the vocal tract must be 331 positive elsewhere and 2) the voicing and pressure parameters 332 must be positive. The area function is a vector function that ${ }_{333}$ describes the transversal shape of the vocal tract. 334

During the vocalization the auditory output of the system 335 is observed along two time windows, the first from 250 to 336 $400 \mathrm{~ms}$ and the second from 650 to $800 \mathrm{~ms}$. The value of each ${ }_{337}$ auditory output is averaged for each time window, the result 338 is a 6-D output signal (two formants and the intonation, hence ${ }_{339}$ three values, per each of the two time windows). In Fig. 3, 340 we reproduce the vocalization representation shown in [9]. To 341 be consistent with the co-articulated nature of speech, only ${ }_{342}$ two perceptual windows are used [1]. However, since only two ${ }_{343}$ portions of the vocalization are considered, a lot of information 344 is lost. For instance, it is shown in [23] the continuum of co- 345 articulated gestures. Therefore, future works should consider 346 studying the continuum of speech gestures and self-structuring 347 of vocalizations.

\section{B. Somatosensory System}

349

In Fig. 3, it is shown that the area function $a_{f}$ is observed ${ }_{350}$ during both perception time windows. The minimal value of 351 the area function $\min \left(a_{f}\right)$ would be zero when the vocal tract 352 is closed at any point and negative values mean that some tis- ${ }_{353}$ sues are overlapped, which does not have physical meaning. 354 However, in some cases it might be interpreted as the tongue 355 being bitten. In other cases it might represent high pressure 356 between the tongue and the palate, which might be interesting ${ }_{357}$ 
358 to the learner in a realistic scenario, where motor constraints 359 are not violated. In general, we made a strong assumption that 360 any motor constraint violation over a threshold is uncomfort361 able or painful. Hence, the average value of $\min \left(a_{f}\right)$ in each 362 perception time window is used to generate a proprioceptive 363 feedback signal $p$ : if the average of $\min \left(a_{f}\right)$ is lower than a 364 threshold for any perception window, then the configuration 365 is evaluated as a undesired collision with $p=1$, and $p=0$ 366 otherwise.

\section{${ }_{367}$ C. Sensorimotor Model}

368 GMMs are linear combinations of multivariate Gaussian 369 distributions that represent clusters of data. They have 370 been previously used to represent nonlinear redundant 371 maps [17], [21], [29] in order to solve the inverse problem 372 of inferring input motor commands from desired sensory out373 puts. GMMs can be learned using an online variant of the 374 expectation-maximization (EM) algorithm in order to learn 375 incrementally from incoming data [30]. Here, the algorithms 376 used to train GMMs are based on the open source tools ${ }^{3}$ 377 associated with [30], and modified according to our problem 378 requirements. The three models in the experimental setup are 379 probabilistic representations in the form of GMMs, obtained 380 using data collected from experiments with the DIVA ear-vocal 381 tract. A detailed explanation of the GMMs training is provided 382 below.

383 We assume that an $n$-dimensional input command space ${ }_{384} X \in \mathbb{R}^{n}$ is mapped to an $m$-dimensional output space $Y \in \mathbb{R}^{m}$, 385 through a transform function $y=f(x)+\varepsilon$, where $y \in Y$, 386 $x \in X$ and $\varepsilon$ is random noise. When a dataset of couples ${ }_{387}(x, y)$ is available, the EM-algorithm is used to obtain a GMM 388 which is defined by the parameters $\left\{\pi_{j}, \mu_{j}, \Sigma_{j}\right\}_{j=1}^{K}$, where $\pi_{j}$, $389 \mu_{j}$, and $\Sigma_{j}$ are, respectively, the prior probability, the distribu390 tion centroid and the covariance matrix of the $j$ th Gaussian, for ${ }_{391} j=1,2, \ldots, K$, being $K$ the number of Gaussian components. 392 From [30], Gaussian mixture regression (GMR) is applied to 393 compute the conditional probability distribution $P(X \mid y)$ in the 394 input space $X$ given a desired output $y$. Once it is computed, 395 the value $x^{*} \in X$ is selected such that it maximizes $P(X \mid y)$.

396 To obtain the input $x$ that maximizes the probability to pro397 duce the output $y$, the GMR process first defines the partitioned 398 vector $z \in X \times Y$, where

399

$$
z=\left(\begin{array}{l}
x \\
y
\end{array}\right)
$$

400 For each Gaussian $j$ in the GMM the partitions

$$
401 \quad \mu_{j}=\left(\begin{array}{l}
\mu_{j}^{x} \\
\mu_{j}^{y}
\end{array}\right) \quad \text { and } \quad \Sigma_{j}=\left(\begin{array}{cc}
\Sigma_{j}^{x} & \Sigma_{j}^{x y} \\
\Sigma^{y x} & \Sigma_{j}^{y}
\end{array}\right)
$$

402 are considered to compute the conditional probability distribu${ }_{403}$ tion $P_{j}(X \mid y) \sim N_{j}\left(\hat{\mu}_{j}, \hat{\Sigma}_{j}\right)$ in the input space $X$ given a desired 404 output $y$, where

$405 \hat{\mu}_{j}=\mu_{j}^{y}+\Sigma_{j}^{y x}\left(\Sigma_{j}^{x}\right)^{-1}\left(x-\mu_{j}^{x}\right), \hat{\Sigma}_{j}=\Sigma_{j}^{y}+\Sigma_{j}^{y x}\left(\Sigma_{j}^{x}\right)^{-1} \Sigma_{j}^{x y}$.

406

\footnotetext{
${ }^{3} \mathrm{http}: / /$ www.calinon.ch/sourcecodes.php
}

Considering that $P(X \mid y)$ is at its maximum when $x=\hat{x}_{j}=\hat{\mu}_{j}, 407$ then a natural selection for $x$ in order to produce $y$ is $\hat{x}_{j}$. But 408 we have $K$ candidates for $x$, hence it is necessary to compute 409

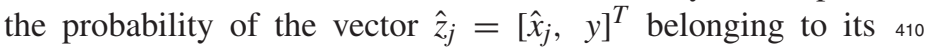
generator Gaussian as

$$
P\left(\hat{z}_{j}\right)=\pi_{j} \frac{1}{\sqrt{(2 \pi)^{K}\left|\Sigma_{j}\right|}} e^{-\frac{1}{2}\left(\left(\hat{z}_{j}-\mu_{j}\right)^{T} \Sigma_{j}^{-1}\left(\hat{z}_{j}-\mu_{j}\right)\right)}
$$

and finally the point $z^{*}=\hat{z}_{j}$ that maximizes $P\left(\hat{z}_{j}\right)$ is selected as 413 the point that better fits the model. In other words, according 414 to our prior knowledge of $f(x), z^{*} \in f(x)$, we infer that the 415 output $y$ is generated by $\hat{x}_{j}$. 416

Taking into account the above for the sensorimotor model, 417 an 18-D motor command space $M$, with $m \in M$, is defined 418 for the vocal tract articulatory configuration. A 6-D auditory 419 output space $S$, with $s \in S$, is also defined, the agent being able ${ }_{420}$ to observe $s$ according to $s=f(m)+\sigma$, where $\sigma \sim N(0,0.01) \quad 421$ is Gaussian noise. The aim is to find a GMM that solves the 422 inverse problem $m=f^{-1}\left(s_{g}\right)$, where $s_{g}$ is an auditory goal. ${ }_{423}$

We define a GMM, $G_{\mathrm{SM}}$, to model the sensorimotor system, 424 with $X=M$ and $Y=S$. Such a model allows computation 425 of the inverse model $P\left(M \mid s_{g}\right)$ using GMR. At the beginning 426 of the experiment, $m$ is selected either, randomly or accord- 427 ing to the interest for initializing the inverse sensorimotor 428 model $m \sim f^{-1}\left(s_{g}\right) \sim P\left(M \mid s_{g}\right)$ around a specific region of ${ }_{429}$ the sensorimotor space. After the initialization stage, the agent 430 starts to select new auditory goals, according to the interest 431 model explained below. In order to reduce memory storage 432 requirements, we consider a generative method for the train- 433 ing stage, which means that the model is trained using the last 434 $N_{\text {SM }}$ samples obtained from experimentation along with $\quad 435$

$$
N_{\text {old }}=\left\lceil\frac{(1-\alpha) N_{\mathrm{SM}}}{\alpha}\right\rceil \text { samples }
$$

generated using $G_{\mathrm{SM}}$, where $\alpha \in[0,1]$ is the forgetting rate. ${ }_{437}$

\section{Interest Model for Auditory Goals}

The interest model for auditory goals endows the learner the 439 ability to select goals that maximize the expected competence 440 progress in order to improve the quality of its sensorimotor 441 model, resulting in better control over it. It is derived from 442 the model proposed in [9]. The competence value for a goal 443 is defined by

$$
c=e^{-\left|s_{g}-s\right|}
$$

where $s_{g}$ is the auditory goal and $s$ is the actual auditory pro- 446 duction after executing a motor command $m \sim P\left(M \mid s_{g}\right)$. To 447 construct the interest model, the auditory goal space is aug- 448 mented with two extra dimensions: 1) the competence $c \in C \quad{ }_{449}$ and 2) time tag $t \in T$. The number of vocalizations $N_{\text {IM }}$ con- 450 sidered to build the interest model is fixed. A GMM, $G_{\mathrm{IM}}$, with 451 $K_{\text {IM }}$ components will be computed from the 8-D data set with 452 $N_{\text {IM }}$ samples of the augmented goal space. To initialize this 453 model, some auditory results from the initialization of $G_{\mathrm{SM}} 454$ are selected as the first auditory goals $s_{g}$.

455

Those Gaussian components in $G_{\mathrm{IM}}$ that, according to the 456 covariance matrices $\Sigma_{j}$, contain goals that will likely increase 457 
458 the competence progressively are considered to build a prob459 abilistic distribution $P(S)$ over the auditory space. In order to 460 build $P(S)$, the components in $G_{\mathrm{IM}}$ are weighted according to 461 their time-competence covariance magnitudes. Thus, $P(S)$ will 462 prioritize goals in regions, where competence is expected to 463 increase. Finally, a sample $s_{g}$ is drawn from $P(S)$ for the next 464 vocalization experiment. Model training is performed every 465 time the agent has performed $n_{\mathrm{IM}}$ experiments, using the last $466 N_{\text {IM }}$ vocalizations.

\section{${ }_{467}$ E. Somatosensory Model}

${ }_{468}$ For the somatosensory model we consider the 18-D motor 469 command space $M$, with $m \in M$, and a new binary propriocep470 tive output space $P=\{0,1\}$, with $p \in P$. If a vocal production 471 leads to undesired contacts, then $p=1$, otherwise $p=0$. A 472 map $g$ is assumed to exist such that $p=g(m)$ and the agent 473 can observe $p$ for each vocal experiment. Thus, it is possible 474 to find a GMM $G_{\mathrm{SS}}$, with $X=M$ and $Y=P$, that allows 475 computation of the probability distribution $P(P \mid m)$ applying ${ }_{476}$ GMR, and determine when a motor command $m$ is likely to 477 lead to an undesired collision in the vocal tract.

478 The inverse sensorimotor model $G_{\mathrm{SM}}$ and the somatosensory 479 model $G_{\mathrm{SS}}$ are initialized together. When an auditory goal $s_{g}$ 480 has been selected, $m$ is computed using $P\left(M \mid s_{g}\right)$. Next, to 481 predict the value of $p, P(P \mid m)$ is used. If the prediction sug${ }_{482}$ gests that $m$ will produce $p=1$ then $s_{g}$ is rejected, otherwise ${ }_{483} s_{g}$ and $m$ are accepted. If $s_{g}$ is rejected, then $G_{\mathrm{IM}}(S)$ is recom${ }_{484}$ puted without considering the Gaussian component in $G_{\mathrm{IM}}$ that 485 generated $s_{g}$, this mechanism decreases the prior of the con${ }_{486}$ flicting Gaussian in $G_{\mathrm{IM}}$. The new $G_{\mathrm{IM}}(S)$ is used to select a ${ }_{487}$ new goal $s_{g}$, and the process is repeated until $s_{g}$ is accepted. ${ }_{488}$ During the agent's life, the model $G_{S S}$ is trained when $G_{S M}$ is 489 trained using the previously described generative mechanism.

\section{F. Self-Exploration Algorithm}

491 The self-exploration architecture with motor constraints 492 self-awareness, first proposed in [8] for ear-vocal tract explo493 ration, is an extended version of [9]. The algorithm associated 494 with the cognitive architecture is shown in Algorithm 1. Our 495 extended self-exploration algorithm with goal babbling and 496 motor constraints self-awareness starts with the learner having 497 no experience in vocalizing. Models $G_{S M}$ and $G_{S S}$ are initial498 ized using random vocalizations with small values around the 499 neutral position of the articulators. The neutral position of the 500 pressure and voicing parameters are set to -0.25 to produce 501 no phonation, whereas for the articulators it is considered 0 , 502 i.e., the rest position. Model $G_{\mathrm{IM}}$ is also initialized.

${ }_{503}$ Then, in line 6 of Algorithm 1 the vocal learner agent selects 504 a goal $s_{g}$ for the next experiment according to the probabilis505 tic distribution $P(S)$ and the motor command $m$ is obtained 506 using the inverse model $G_{\mathrm{SM}}$ in line 7. The main feature of 507 this algorithm, different from similar architectures, is that in ${ }_{508}$ line $8 P(P \mid m)$ provides a prediction for $p$ that indicates if 509 the selected motor command is likely to produce an undesired 510 collision. From line 9 to 11 , if $p \approx 1$, the goal is rejected 511 and the probabilistic distribution $P(S)$ is updated, ignoring the

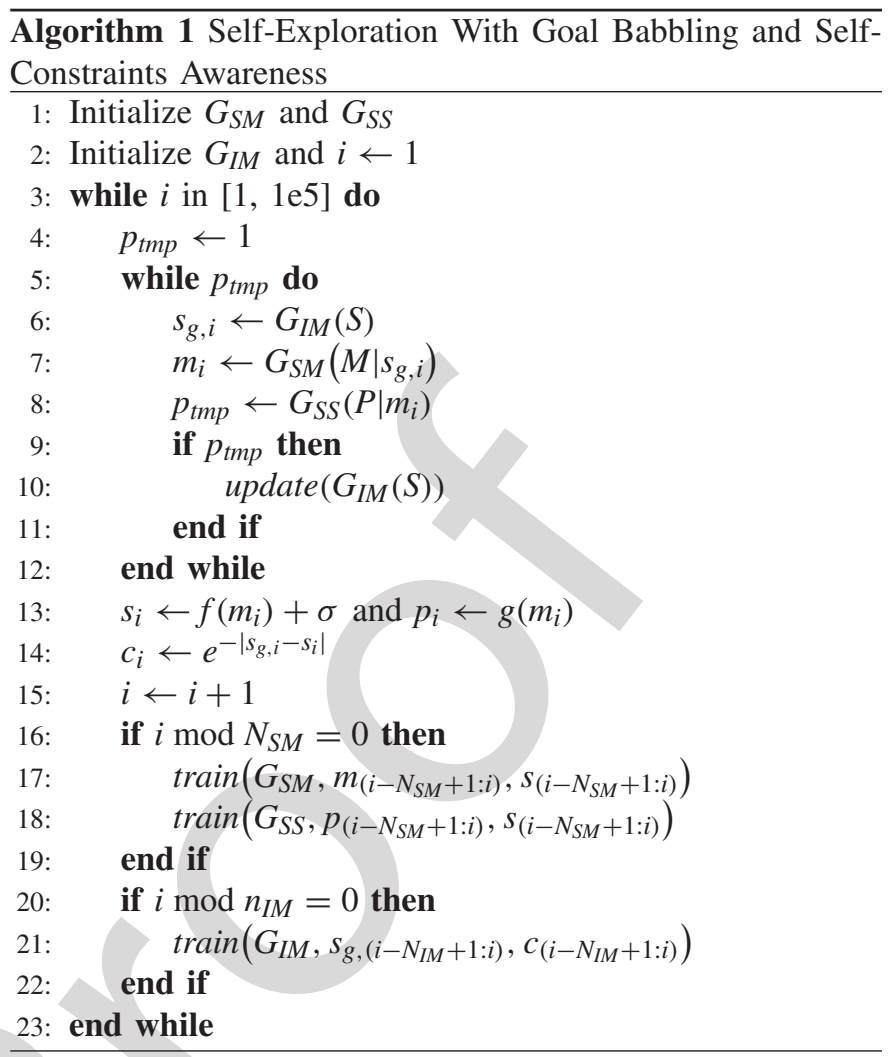

Gaussian component in $G_{\mathrm{IM}}$ that generated $s_{g}$ and the algo- 512 rithm goes back to line 6 . Otherwise, $p \approx 0$, both, $s_{g}$ and $m$ are ${ }_{513}$ accepted. Next, the motor command is executed with the vocal 514 tract and the agent observes $s$ and $p$ in line 13. In line 14, the 515 learner evaluates the competence value $c$. It also checks if we 516 are at the end of a learning episode, so models $G_{\mathrm{SM}}, G_{\mathrm{SS}}$, and ${ }_{517}$ $G_{\mathrm{IM}}$ are updated in lines 17,18 , and 21 , respectively. To pro- 518 vide objective evaluation elements, some experiments without 519 considering the somatosensory model for choosing goals are 520 also presented. In this later case, $s_{g}$ is always accepted, thus 521 line 4 is substituted with $p_{\text {tmp }}=0$ in Algorithm 1 .

\section{EXPERIMENTAL RESULTS}

523

Eighteen independent simulations using Algorithm 1 were ${ }_{524}$ run. All simulations consisted of half a million of vocaliza- 525 tions, including an initial vocalization set of 1000 random 526 samples. Nine different random seeds were considered to ${ }_{527}$ generate the same number of motor command sets from a ${ }_{528}$ uniform distribution. The limits for those motor commands ${ }_{529}$ related to the vocal tract articulators were $[-1,1]$, whereas 530 for motor commands related to the phonation parameters were ${ }_{531}$ $[0,0.7]$. Each set was used twice to initialize simulations of ${ }_{532}$ Algorithm 1, first without using the somatosensory model and ${ }_{53}$ second with it.

Considering as a reference the parameters used for simula- 535 tions in [8] and [9], a few variations in their values were tested. 536 First, when values for $K_{\mathrm{SM}}$ or $K_{\mathrm{SS}}$ are increased the inference ${ }_{537}$ error decreases slightly but the computation time grows con- 538 siderably. On the other hand if these values are decreased, the ${ }_{539}$ inference error increases considerably. Second, if the training 540 


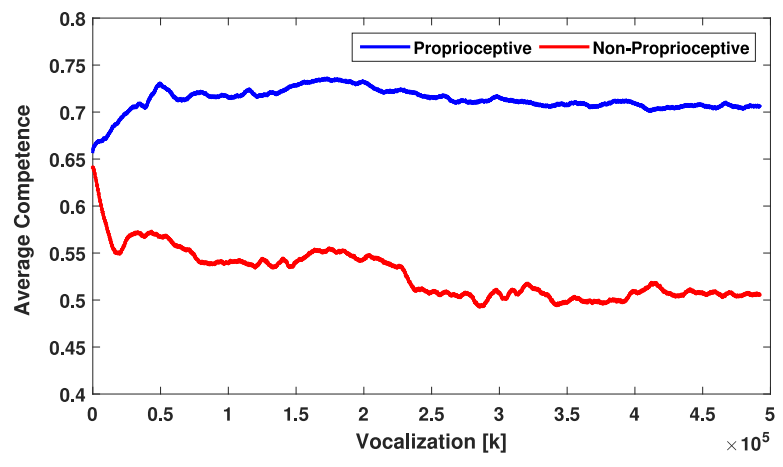

Fig. 4. Mean competence evolution during simulations using Algorithm 1 for nine different initialization data sets. Moving average of 5000 samples are considered to filter the results of each simulation. Results are shown in the case of proprioceptive and nonproprioceptive agents.

541 steps are increased for the somatosensory model and the senso542 rimotor model, then the training computational time increases ${ }_{543}$ as $N_{\text {old }}$ in (6) increases proportionally, but no improvement 544 is obtained in the inference error. However, if these values 545 are decreased beyond the values used in [9], the mean infer${ }_{546}$ ence error increases. Third, when $\alpha_{\mathrm{SM}}$ and $\alpha_{\mathrm{SS}}$ are larger than ${ }_{547} 0.1$ the competence progress is slower. Finally, the parame548 ters linked to the interest model allow a wider range of values 549 to be chosen obtaining similar results. For $K_{\text {IM }}$ we observed 550 that values greater than or equal to 12 worked similarly, but ${ }_{551}$ smaller values negatively impacted the competence progress. 552 Thus, the main parameters for all the simulations were kept 553 as in [8] and [9] as they performed better than other sim554 ulations in terms of exploration results and simulation time. 555 Summarizing, values were set to $K_{\mathrm{SM}}=28, N_{\mathrm{SM}}=400$, ${ }_{556} K_{\mathrm{SS}}=28, K_{\mathrm{IM}}=12, N_{\mathrm{IM}}=4800, n_{\mathrm{IM}}=12$, and the con557 tinuous sampling time used for the DIVA ear-vocal tract was ${ }_{558} t_{s}=10 \mathrm{~ms}$. The forgetting rate parameter $\alpha_{\mathrm{SM}}$ for $G_{\mathrm{SM}}$ starts 559 from 0.1 and decreases logarithmically to 0.05 after half a 560 million of vocalizations. On the other hand, $\alpha_{S S}$ for $G_{S S}$ was ${ }_{561}$ chosen to be 0.05 through the whole simulation. ${ }^{4}$

562 During the simulation, $G_{\mathrm{SM}}$ and $G_{\mathrm{SS}}$ are initialized as indi563 cated in line 1 of Algorithm 1 with the initial motor command 564 sets. Then, all the initial phonatory productions are used as 565 auditory goals to initialize the interest model $G_{\mathrm{IM}}$ as indicated 566 in line 2 of Algorithm 1. In this stage, $G_{S M}$ is used to infer the 567 motor commands that will likely produce the initial auditory 568 goals. These commands are executed without considering the 569 proprioceptive prediction $p$.

\section{A. On Competence and Contacts}

571 First of all, Fig. 4 represents the evolution of the compe572 tence parameter $c$ in (7) for self-generated auditory goals. To 573 obtain this plot, first the result of each simulation is filtered 574 using a 5000 samples moving average window. Next, simu575 lations are divided into two general groups: 1) proprioceptive 576 agents and 2) nonproprioceptive agents. Finally, the mean of

\footnotetext{
${ }^{4}$ Supplementary downloadable material provided by the authors is available at https://dx.doi.org/10.6084/m9.figshare.c.3676645.v1. After each experiment, 20 random samples from the last 1000 vocalizations were drawn to generate videos with audio. Videos of the experiments 1,5, and 9 are provided.
}

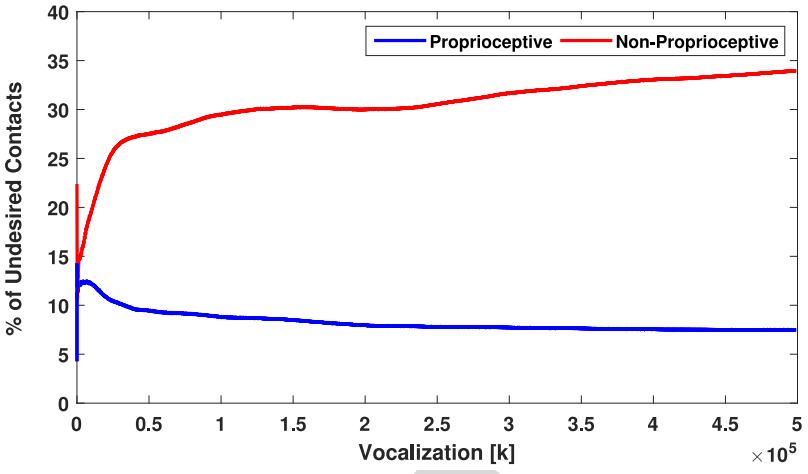

(a)

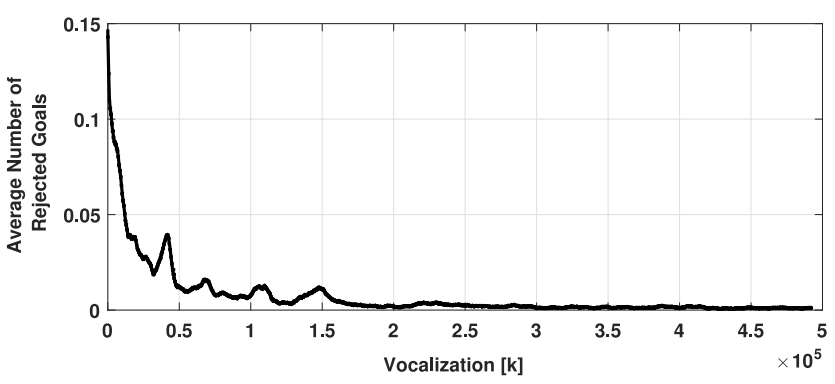

(b)

Fig. 5. Algorithm 1 simulation results. (a) Mean percentage of vocalizations producing undesired collisions considering all the simulated agents. Agents are grouped by proprioceptive and nonproprioceptive. The results of each agent are prefiltered considering a 5000 samples moving average. (b) Mean number of rejected goals using the proprioceptive prediction and considering all the simulated proprioceptive agents. The results of each agent are prefiltered considering a 5000 samples moving average.

TABLE I

Results Considering All Data From Exploration

\begin{tabular}{|c|c|c|c|c|c|c|}
\hline \multirow{2}{*}{ Experiment } & \multicolumn{3}{|c|}{ Non-Proprioceptive } & \multicolumn{3}{|c|}{ Proprioceptive } \\
\cline { 2 - 7 } & Vol. & mean $(c)$ & \% Contacts & Vol & mean $(c)$ & \% Contacts \\
\hline \hline 1 & 0.58 & 0.50 & $41.67 \%$ & 0.49 & 0.80 & $3.42 \%$ \\
\hline 2 & 0.47 & 0.50 & $37.50 \%$ & 0.49 & 0.76 & $3.88 \%$ \\
\hline 3 & 0.49 & 0.50 & $43.53 \%$ & 0.43 & 0.61 & $5.28 \%$ \\
\hline 4 & 0.47 & 0.54 & $30.83 \%$ & 0.52 & 0.73 & $9.03 \%$ \\
\hline 5 & 0.47 & 0.54 & $29.17 \%$ & 0.44 & 0.68 & $15.55 \%$ \\
\hline 6 & 0.56 & 0.59 & $23.01 \%$ & 0.52 & 0.70 & $8.18 \%$ \\
\hline 7 & 0.49 & 0.55 & $30.86 \%$ & 0.41 & 0.71 & $6.76 \%$ \\
\hline 8 & 0.57 & 0.54 & $35.49 \%$ & 0.43 & 0.68 & $8.57 \%$ \\
\hline 9 & 0.49 & 0.50 & $32.42 \%$ & 0.63 & 0.74 & $6.74 \%$ \\
\hline \hline Average & 0.51 & 0.53 & $33.83 \%$ & 0.48 & 0.71 & $7.49 \%$ \\
\hline Min & 0.47 & 0.50 & $23.01 \%$ & 0.41 & 0.61 & $3.42 \%$ \\
\hline Max & 0.58 & 0.59 & $43.53 \%$ & 0.63 & 0.80 & $15.55 \%$ \\
\hline
\end{tabular}

Note: Experiments with different vocalization initial sets for Proprioceptive and NonProprioceptive Agents. The volume of a the convex-hull described by the explored data, the mean value for the competence $c$, and the final percentage of contacts along the simulations are shown.

all the filtered results for each group is computed. The same 577 mechanism is considered to obtain the percentage of contacts 578 observed in Fig. 5(a).

Tables I and II show the volume of a convex hull cov- 580 ering the explored auditory region. They also display the ${ }_{581}$ mean competence and the percentage of undesired contacts 582 at the end of the simulation. First, in Table I, descriptors are ${ }_{583}$ computed considering all the vocalization during each simula- 584 tion. Second, in Table II, figures were computed considering 585 
TABLE II

RESUlTS WiTHOUT CONSIDERING VOCALIZATIONS WITH UNDESIRED CONTACTS

\begin{tabular}{|c||c|c|c|c|}
\hline \multirow{2}{*}{ Experiment } & \multicolumn{2}{c|}{ Non-Proprioceptive } & \multicolumn{2}{c|}{ Proprioceptive } \\
\cline { 2 - 5 } & Vol. & mean $(c)$ & Vol. & mean $(c)$ \\
\hline \hline 1 & 0.48 & 0.69 & 0.39 & 0.81 \\
\hline 2 & 0.38 & 0.67 & 0.39 & 0.78 \\
\hline 3 & 0.37 & 0.71 & 0.33 & 0.63 \\
\hline 4 & 0.40 & 0.65 & 0.42 & 0.77 \\
\hline 5 & 0.36 & 0.66 & 0.37 & 0.76 \\
\hline 6 & 0.44 & 0.67 & 0.42 & 0.74 \\
\hline 7 & 0.36 & 0.69 & 0.32 & 0.74 \\
\hline 8 & 0.45 & 0.67 & 0.35 & 0.71 \\
\hline 9 & 0.38 & 0.64 & 0.49 & 0.78 \\
\hline \hline Average & 0.40 & 0.67 & 0.39 & 0.75 \\
\hline Min & 0.36 & 0.64 & 0.32 & 0.63 \\
\hline Max & 0.48 & 0.71 & 0.49 & 0.81 \\
\hline
\end{tabular}

Note: Experiments with different vocalization initial sets for Proprioceptive and Non Proprioceptive Agents. The volume of a the convex-hull described by the explored data and the mean value for the competence $c$ along the simulations are shown.

586 vocalizations without undesired collisions. Convex hulls pro${ }_{587}$ vide an insight regarding the size of the explored regions in 588 the auditory-space. They are computed considering formant 589 frequency dimensions $F_{11}, F_{21}, F_{12}$, and $F_{22}$.

590 In Fig. 4, results suggest that proprioceptive agents perform 591 better than those which are not endowed with proprioception. 592 We observe that at the beginning of the exploration the mean 593 average competence is very similar for both groups. However, 594 after the initialization the nonproprioceptive agents suffer an 595 important decrement of the competence, which also coincides 596 with a significant increment of the percentage of contacts in 597 Fig. 5(a). Table I also confirms the expected results accord598 ing to our hypothesis: using proprioceptive feedback drives 599 artificial agents to produce significantly fewer undesired con600 tacts and also increases the competence to reach self-generated 601 auditory goals.

602 Some observers might ask the reason of high competence 603 values at the beginning of the simulations. We argue that it is 604 an expected result as the competence computation begins when ${ }_{605} G_{\mathrm{IM}}$ is initialized with self-generated goals drawn from the ini606 tial auditory productions of the agent. In other words, $G_{\mathrm{SM}}$ and ${ }_{607} G_{S S}$ models are initialized around a set of initial vocalizations 608 and auditory productions. The initial auditory productions are 609 selected as auditory goals, then motor commands are computed 610 with a sensorimotor model that represents very well those ini611 tialization samples. Later, as the agent explores the auditory 612 space and it moves toward farther regions from those of initial613 ization, the competence values might slightly decrease. This 614 is due to the incremental learning of probabilistic models and ${ }_{615}$ depends on the values assigned to the forgetting rates $\alpha_{\mathrm{SM}}$ and ${ }_{616} \alpha_{\mathrm{SS}}$. If these forgetting rates are close to zero, then the agent 617 is less prone to update its knowledge when new data are far 618 from the current knowledge. On the other hand, if the forget619 ting rates are high, then the agent will adapt its model to the 620 new data very fast but also it will forget faster its previous 621 knowledge since it is not reinforced.

622 We also argue that one of the reasons the propriocep623 tive agents perform better is the null competence produced 624 by nonphonatory vocalizations. The nonproprioceptive agent 625 produces much more undesired contacts (four times more

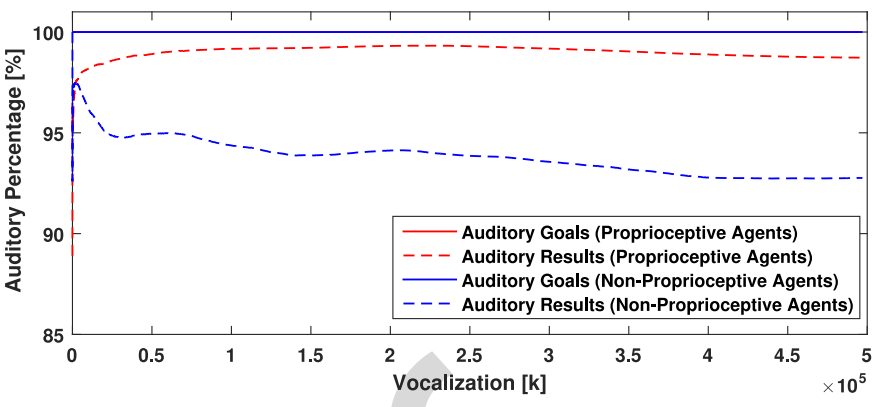

Fig. 6. Mean percentage of vocalizations producing phonatory result along all the simulations per each group, proprioceptive and nonproprioceptvie agents. The percentage of phonatory auditory goal is also shown per each group. Note: red and blue solid lines are overlapped.

undesired contacts on average) and, therefore, has more non- 626 phonatory vocalizations as corroborated in Fig. 6. Fig. 6 was 627 obtained using the same procedure than Fig. 4. It shows the 628 mean percentage of phonatory goals and actual phonatory 629 vocalizations considering all the simulations per each group 630 of artificial agents. In general, all the auditory goals through 631 the simulations are phonatory. The reason is that nonphonatory 632 goals become uninteresting very early in the artificial agent's 633 life as they are very easy to be produced. Thus, all those 634 nonphonatory vocalizations which produce null competence 635 impact negatively the average competence. We also might 636 think about all those nonphonatory vocalizations as a waste 637 of energy during the exploration. Knowing which regions of 638 the motor space are leading to collisions might be a relevant 639 knowledge for the agent. However, as the nonproprioceptive 640 agents keep exploring conflicting regions, the proprioceptive 641 agents avoid exploitation of these regions due to their ability to 642 predict somatosensory results from a given motor command. 643

Additionally, discussion about the tradeoff between explo- 644 ration and exploitation can be detailed. We argue that pro- 645 prioceptive agents show a better performance with respect to 646 exploitation, as agents avoid exploring uninteresting regions 647 with high number of contacts. In other words, propriocep- 648 tion, and in general constraint awareness, contributes to the 649 agent finding regularities faster and then fosters specialization 650 in regions of the auditory space, where the agent competence 651 to reach self-generated goals is higher. It is worth mentioning 652 that we are aware that it is also important to include the social 653 factor in the learning development of the artificial agent, in 654 order to better understand the role of proprioception in social 655 learning. In social learning, exploration is not just driven by 656 the progress in competence and discovery of constraints, but 657 also by the relevance of auditory goals for socialization pur- 658 poses. These studies leading to more exploring behaviors is 659 left for future work.

660

Furthermore, Fig. 5(b) shows the mean number of goals 661 rejected by the proprioceptive mechanism, represented in 662 lines 5-12 of Algorithm 1. In this plot, we prefilter the results 663 for proprioceptive agents considering a 5000 samples mov- 664 ing average for visualization purposes. It can be observed in 665 Fig. 5(b) that in general the proprioceptive mechanism is more 666 active at the beginning of the simulations presumably due to 667 the quantity of contacts along the initial set of vocalizations. 668 
${ }_{669}$ Thus, we might deduce that proprioception prevents the agent 670 from further exploration in regions that are producing unde671 sired contacts especially in the early stages. In the next, we 672 introduce some figures in order to show the implications over 673 the shape of the explored auditory region when proprioception 674 is considered.

\section{B. On Explored Regions}

676 Regarding the volume of the explored region, Table I 677 indicates that the ratio of average volume of convex hulls 678 described by the explored regions in the frequency space is $6790.51 / 0.48$ between the nonproprioceptive and proprioceptive 680 agents, whereas the ratio of the mean competence is $0.53 / 0.71$. 681 In other words, whereas proprioceptive agents explore a $5.88 \%$ 682 tighter region than the nonproprioceptive, their performance 683 is $25.35 \%$ better than the later ones. On the other hand, 684 Table II considers only the vocalizations without undesired 685 contacts. A shrinkage of the convex hulls is observed, the 686 ratio of average volumes is, in this case, $0.40 / 0.39$ while 687 the mean competence ratio is $0.67 / 0.75$. From these numbers 688 we observe that, in general, the competence to vocalizations 689 without undesired contacts is higher for both kinds of agents. 690 However, regarding competence the proprioceptive agents still ${ }_{691}$ perform $11.94 \%$ better than the nonproprioceptive agents.

692 Based on Table I, we selected three different initial sets ${ }_{693}$ given their simulation results in order to produce Figs. 7-9. 694 First, we select initial set 1, as it performs better in terms 695 of competence when proprioception is considered. Second, to 696 contrast with initial set 1 we select initial set 5 , as its proprio697 ceptive agent performs the worst with regards to the percentage 698 of undesired contacts. Finally, we select initial set 9, as its 699 proprioceptive agent produced the largest convex hull volume. 700 Figs. 7-9 show some projections of vocalizations distribu701 tion maps of the auditory productions generated along the 702 simulation. Points in the plots are colored according to the 703 percentage of undesired contacts produced in its neighbor704 hood. Specifically, three projections are shown for each of 705 the selected sets of initial vocalizations. Projection $F_{1,1} F_{2,1}$ 706 represents the auditory fingerprint of the vocalizations in the 707 first perceptual window. Projections $F_{1,2} F_{2,2}$ is similar to the 708 first projection but for the second perceptual window. Finally, 709 projection $I_{1} I_{2}$ represents the value of the intonation parameter 710 in the first perceptual window against the same parameter in 711 the second perceptual window.

712 Distributions in Figs. 7-9 indicate that the intonation param713 eter projection $I_{1} I_{2}$ is the most influenced sensory-output due 714 to the proprioceptive feedback. Recall that $I_{1}$ and $I_{2}$ depend 715 on the average audibility of the vocalization which is null in 716 two cases: 1) when the voicing parameters are lower than zero 717 or 2) when the area function of the vocal tract is nonpositive 718 elsewhere. Therefore, keeping in mind the latter case, those 719 vocalizations producing an intonation parameter (either $I_{1}$ or $720 I_{2}$ ) lower than one indicate that a contact has likely occurred. ${ }_{721}$ If a contact occurs, there are two possible results, the average 722 of the minimum value of the area function might be nega723 tive or not. If it is negative, then the contact is classified as an 724 undesired contact and the proprioceptive signal takes the value
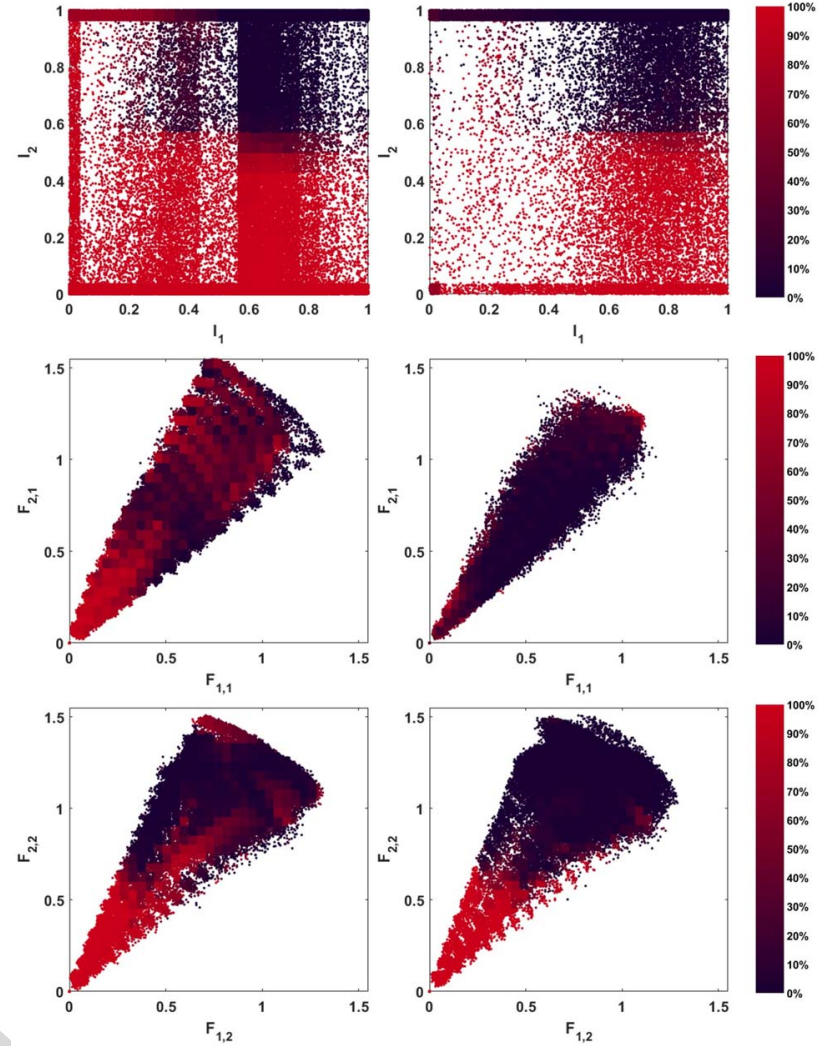

Fig. 7. Projections of vocalizations distribution along simulations using initial set 1 with Algorithm 1. Results for nonproprioceptive agent (left) and proprioceptive agent (right). Points are colored according to the total percentage of undesired contacts in their neighborhood.

one. Thus, having both values lower than one at the same time 725 is even more likely to produce undesired contacts. That is the 726 reason proprioceptive agents explore less intensively the mid- ${ }_{727}$ dle of the region in the intonation space. However, we argue 728 that in spite of the low density of vocalizations in that region, 729 proprioceptive agents succeed in finding more vocalizations 730 that produces nonconflicting articulatory configurations in that 731 region. For instance, looking at the projections in Fig. 9, the 732 proprioceptive agent almost covers all the intonation space ${ }_{733}$ with low density of contacts.

Moreover, comparing proprioceptive and nonproprioceptive 735 agents in Figs. 7-9, we observe that the area of the explored 736 regions varies slightly due to the proprioceptive mechanism. ${ }_{737}$ This fact is supported by Tables I and II. In general, in most ${ }_{738}$ of the cases using the proprioceptive feedback results in a ${ }_{739}$ slightly smaller explored region but this is not a conservative 740 fact. For instance, Table I indicates that the convex hull vol- 741 umes described in the auditory space by the experiments 2, 742 4 , and 9 were larger when proprioception was considered. In 743 general, besides a certain degree of randomness due to our 744 probabilistic approach, we argue that there are three main ele- 745 ments that determine the shape of the explored region: 1) the 746 initial set of vocalizations; 2) evolution of competence; and 747 3) proprioception.

748

Regarding the initial set, our criteria to choose random 749 vocalizations close to the neutral positions produces rich sets 750 of phonatory vocalizations, either with contacts or without. 751 

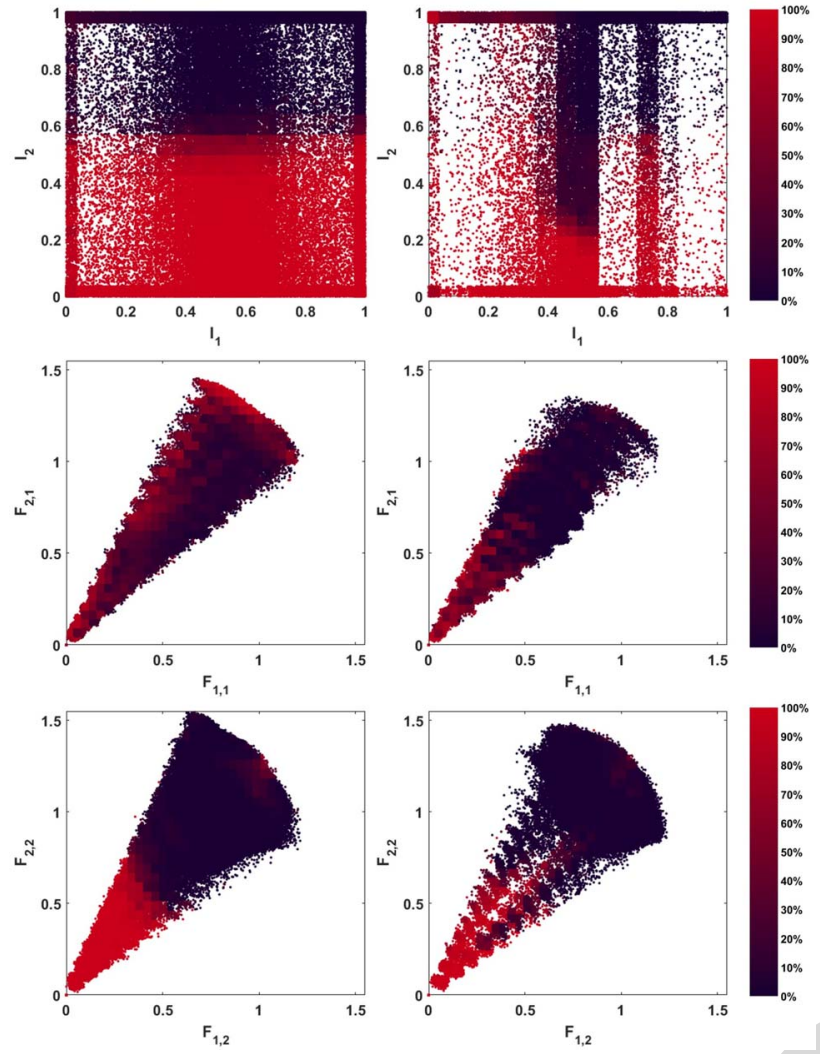

Fig. 8. Projections of vocalizations distribution along simulations using initial set 5 with Algorithm 1. Results for nonproprioceptive agent (left) and proprioceptive agent (right). Points are colored according to the total percentage of undesired contacts in their neighborhood.

752 As we are working with self-generated goals, the agent is 753 expected to be very good at the beginning at reaching goals 754 when auditory goals are close to the initialization region. In 755 the case of nonproprioceptive agents, the only parameter that 756 drives the exploration is the evolution of competence, which 757 is why we observe plenty of areas with a huge amount of 758 undesired contacts in the plots of nonproprioceptive agents 759 with respect to the proprioceptive agents. Furthermore, we 760 observe that proprioception might lead toward two different 761 situations: 1) an unexplored auditory region or 2) explored 762 region but with nonconflicting articulatory configurations. For 763 instance, in Fig. 7 , specifically in the projection $F_{1,1} F_{2,1}$, we ${ }_{764}$ observe that in general the nonproprioceptive agents produce 765 a lot of undesired contacts over almost the whole explored 766 region. On the contrary, the proprioceptive agent explores a 767 smaller region over the same projection, however it achieves 768 a considerably lower density of undesired contacts; results also 769 supported by the convex hull volume displayed in Table I. In 770 addition, projection $F_{1,2} F_{2,2}$ shows similar explored regions 771 for both agents. Indeed, the proprioceptive agent explored a 772 wider region in that projection and was capable of finding non773 conflicting vocalizations for some of the regions, where the 774 nonproprioceptive agent produces a lot of undesired contacts. 775 We observe, in general, for all the agents in Figs. 7-9, that 776 producing auditory results for the projection $F_{1,2} F_{2,2}$ close to 777 the origin is hard without producing contacts.
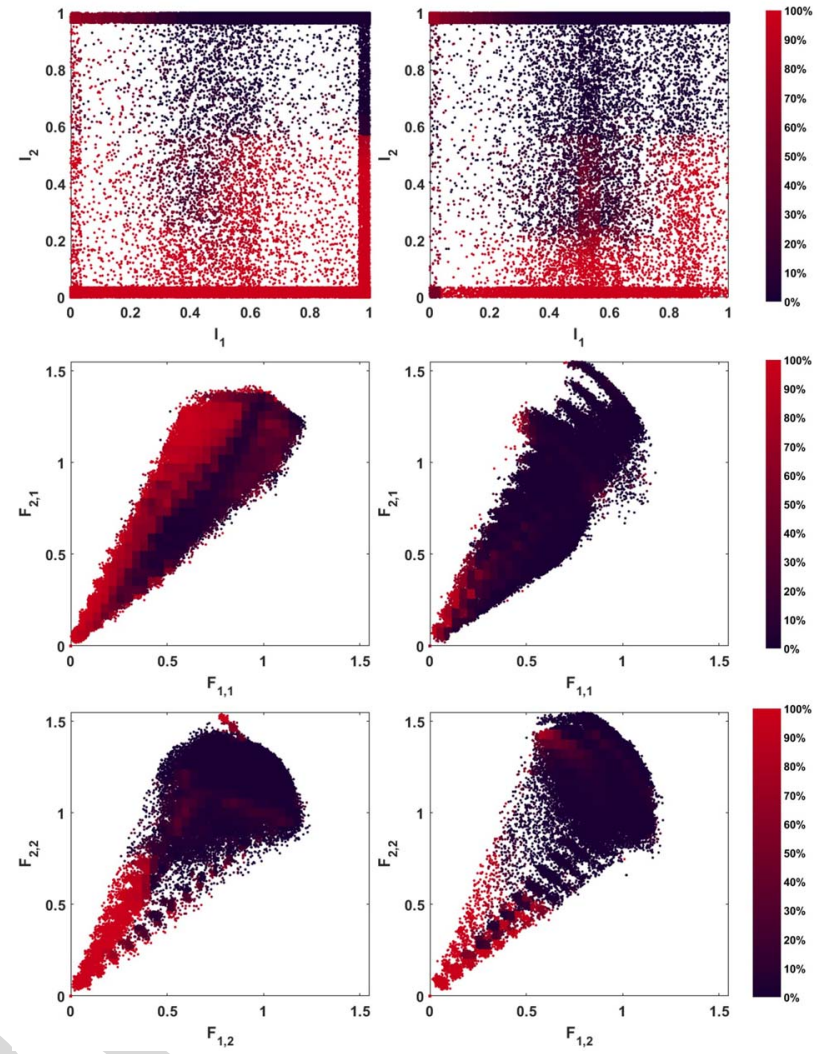

Fig. 9. Projections of vocalizations distribution along simulations using initial set 9 with Algorithm 1. Results for nonproprioceptive agent (left) and proprioceptive agent (right). Points are colored according to the total percentage of undesired contacts in their neighborhood.

In Fig. 8, corresponds to the agent with the worst results 778 using proprioception regarding the number of undesired con- 779 tacts, the projection $F_{1,1} F_{2,1}$ indicates that the proprioceptive 780 agent has explored a smaller region than the nonproprioceptive 781 agent. However, if we observe the boundaries of the explored 782 region with proprioception, they coincide with regions where 783 the nonproprioceptive agent produces a high amount of unde- 784 sired contacts. Thus, the proprioceptive mechanism does not 785 allow the proprioceptive agent to exploit those regions. In spite 786 of less exploration, we observe that in the explored region 787 where both agents intersect, the proprioceptive agent produces 788 less undesired contacts. Looking at Table II, the results for 789 the agent corresponding to Fig. 8 (experiment 5), we observe 790 that the explored regions with and without proprioception are 791 described by convex hulls with similar volume. This suggests 792 that the conflicting region explored by the nonpropriocep- 793 tive agent prevents the agent from exploiting regions without 794 undesired contacts. Thus, the agent achieve lower competence 795 values over the latter regions. On the other hand, the propri- 796 oceptive agent avoids conflicting regions, in consequence it 797 produces $15 \%$ less contacts and achieve a higher competence 798 average.

799

In addition, looking at the projection $F_{1,1} F_{2,1}$ Fig. 9, we 800 observe that the proprioceptive agent explores a larger region. 801 Moreover the density of contacts along the explored region is 802 significantly lower. This is also supported by the numerical 803 results in Tables I and II. On the other hand, in the projection 804 


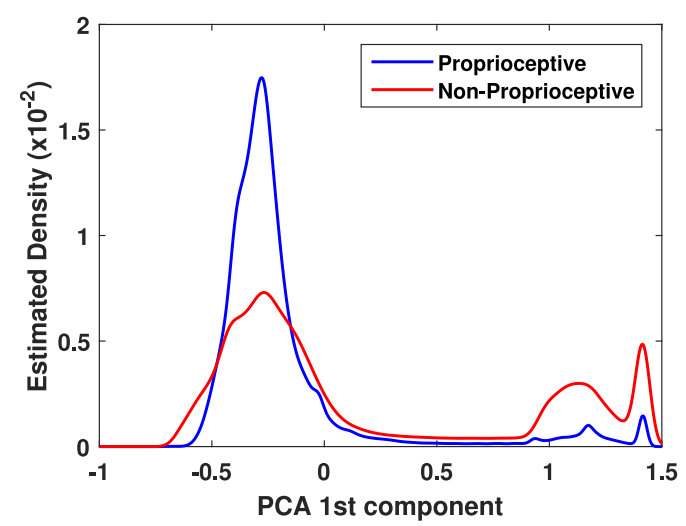

Fig. 10. Density distribution computed using Gaussian-kernels over all the data obtained along the simulations considering the first principal component with a variance contribution ratio of 0.68 .

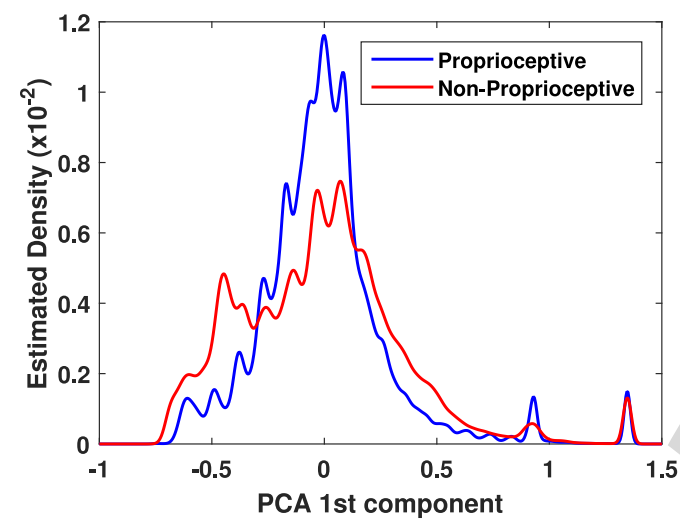

Fig. 11. Density distribution computed using Gaussian-kernels over the data, excluding vocalizations with undesired contacts, obtained along the simulations considering the first principal component with a variance contribution ratio of 0.61 .

$805 F_{1,2} F_{2,2}$ the exploration close to the origin of that projection 806 is less intensive in the proprioceptive agent, which reinforces 807 the previous observation of the difficulties to produce audi808 tory results in that region without contacts, similar results are 809 observed in Fig. 8. Future work also must focus in the study 810 of what is happening in that region and how relevant it is to 811 language, as well as modify the system accordingly.

${ }_{812}$ Finally, in order to observe the differences between the 813 vocalization distributions obtained using the different explo814 ration algorithms, we perform a sample density analysis over 815 the formant frequency dimensions. In order to make the results 816 easier to visualize we perform a principal component analysis 817 (PCA) procedure. We consider analyzing the data twice, first, 818 considering all the data collected along the exploration and 819 second, without considering the vocalizations with undesired 820 contacts. The PCA is done considering the dimensions $F_{1,1}$, $821 F_{2,1}, F_{1,2}$, and $F_{2,2}$, the data of all the 18 simulations are con822 catenated and used to perform the PCA. The PCA considering ${ }_{823}$ all the samples is performed and the first component is kept, 824 which contributes to the variance with a ratio of 0.61 . A sec825 ond PCA is performed considering only the non conflicting 826 vocalizations, again only the first component is kept, since it 827 contributes to the variance with a ratio of 0.68 . Once PCA transforms 4-D data into 1-D data, kernel-distribution estima- 828 tion is performed using Gaussian-kernels according to [31] for 829 the proprioceptive and nonproprioceptive cases.

830

In Figs. 10 and 11, we can observe the density distributions 831 obtained separately with all the proprioceptive and the nonpro- 832 prioceptive agents. First in Fig. 10, the distribution considering 833 all the data obtained from all the experiments is shown. In gen- 834 eral, it is observed that the agents explored similar regions, 835 but with different intensity. In Fig. 11, we observe the distri- 836 bution of the first component obtained from the PCA when ${ }_{837}$ only nonconflicting vocalizations are considered. In the latter 838 case it is observed that regarding the regions which are of 839 interest, in other words the regions where physical constraints 840 are not violated, both kinds of agents explore with a simi- 841 lar density shape, which means that even though both agents 842 explore similar interesting regions, the proprioceptive agents ${ }_{843}$ achieve in general higher competence.

\section{CONCLUSION}

845

An application of active learning techniques applied to the 846 study of vocal exploration considering motor constraints has 847 been introduced. It has been presented as an intrinsically moti- 848 vated sensorimotor self-exploration architecture with motor 849 constraints self-awareness. Constraints awareness is achieved 850 by providing a proprioceptive mechanism which endows an 851 artificial agent with the capacity to autonomously generate a 852 somatosensory model. This model is then used to predict the 853 consequences of a motor action and to avoid its execution if 854 it is expected to generate an undesired proprioceptive result. 855

The proprioceptive mechanism improved the quality of 856 learning according to a competence function. However, we ${ }_{857}$ observe a tradeoff between exploration and exploitation, 858 predominantly nonproprioceptive agents achieve greater explo- 859 ration in the auditory space. In contrast, we observe a more 860 intensive exploitation in interesting regions driving to the 861 higher competence values achieved by proprioceptive agents. 862 In general, vocal-auditory spaces are high dimensional redun- 863 dant spaces, thus an auditory output may be produced by 864 different articulatory configurations. Some of these articula- 865 tory configurations may lead to undesired contacts. Hence, we 866 argue that sensorimotor redundancy is reduced when proprio- 867 ception is included in the system allowing the agent to focus on 868 exploitation of nonconflicting vocalizations. In consequence, 869 the sensorimotor model generated through the exploration does 870 not include conflicting regions, where constraint violations are 871 likely to happen. For that reason, sensorimotor models achieve 872 better fitting to the regions of interest where constraints are 873 met. In this way, we showed how sensorimotor exploration, 874 and in general sensorimotor knowledge, can be shaped by 875 constraints.

Regarding the advance toward vocal exploration, we have 877 showed the suitability of the presented architecture to learn 878 vocal spaces in interesting and less redundant regions as chil- 879 dren might do. However, in order to continue our research 880 on early vocal development, we must study in greater depth 881 the first period of vocalization development. A deeper anal- ${ }_{882}$ ysis of the learning processes underlying the nonauditory 883 
884 development related to mastication, deglutition, and crying 885 from the cognitive and developmental perspectives should be 886 completed in order to generate more complex somatosensory 887 architectures. Finally, the next step of this paper should be 888 directed toward the self-structuring of vocalization and social 889 learning.

\section{REFERENCES}

[1] P. K. Kuhl, "Early language acquisition: Cracking the speech code," Nat. Rev. Neurosci., vol. 5, no. 11, pp. 831-843, 2004.

2] M. Asada et al., "Cognitive developmental robotics: A survey," IEEE Trans. Auton. Mental Develop., vol. 1, no. 1, pp. 12-34, May 2009.

[3] R. Pfeifer, M. Lungarella, and F. Iida, "Self-organization, embodiment, and biologically inspired robotics," Science, vol. 318, no. 5853, pp. 1088-1093, 2007.

[4] R. Pfeifer and C. Scheier, Understanding Intelligence. Cambridge, U.K.: MIT Press, 1999.

[5] D. A. Cohn, Z. Ghahramani, and M. I. Jordan, "Active learning with statistical models," J. Artif. Intell. Res., vol. 4, no. 1, pp. 129-145, 1996.

[6] S. Thrun, "Exploration in active learning," in Handbook of Brain Science and Neural Networks, 1995, pp. 381-384.

[7] C. Moulin-Frier and P.-Y. Oudeyer, "Exploration strategies in developmental robotics: A unified probabilistic framework," in Proc. Int. Conf. Develop. Learn. (ICDL/Epirob), Osaka, Japan, 2013, pp. 1-6.

[8] J. M. Acevedo-Valle, C. Angulo, N. Agell, and C. Moulin-Frier, "Proprioceptive feedback and intrinsic motivations in early-vocal development," in Proc. 18th Int. Conf. Catalan Assoc. Artif. Intell., 2015, pp. $9-18$.

[9] C. Moulin-Frier, S. M. Nguyen, and P.-Y. Oudeyer, "Self-organization of early vocal development in infants and machines: The role of intrinsic motivation," Front. Psychol., vol. 4, pp. 1006-1025, Jan. 2014 doi: 10.3389/fpsyg.2013.01006.

10] F. H. Guenther, S. S. Ghosh, and J. A. Tourville, "Neural modeling and imaging of the cortical interactions underlying syllable production," Brain Lang., vol. 96, no. 3, pp. 280-301, 2006.

[11] A. S. Warlaumont, G. Westermann, E. H. Buder, and D. K. Oller, "Prespeech motor learning in a neural network using reinforcement," Neural Netw., vol. 38, pp. 64-75, Feb. 2013.

12] B. J. Kröger, J. Kannampuzha, and C. Neuschaefer-Rube, "Towards a neurocomputational model of speech production and perception," Speech Commun., vol. 51, no. 9, pp. 793-809, 2009.

[13] I. S. Howard and P. Messum, "Modeling the development of pronunciation in infant speech acquisition," Motor Control, vol. 15, no. 1, pp. 85-117, 2011.

[14] M. Rolf, J. J. Steil, and M. Gienger, "Goal babbling permits direct learning of inverse kinematics," IEEE Trans. Auton. Mental Develop., vol. 2, no. 3, pp. 216-229, Sep. 2010.

15] A. Baranes and P.-Y. Oudeyer, "Active learning of inverse models with intrinsically motivated goal exploration in robots," Robot. Auton. Syst., vol. 61, no. 1, pp. 49-73, 2013.

16] C. Moulin-Frier and P.-Y. Oudeyer, "Learning how to reach various goals by autonomous interaction with the environment: Unification and comparison of exploration strategies," in Proc. 1st Multidiscipl. Conf. Reinforcement Learn. Decis. Making (RLDM), Princeton, NJ, USA, Oct. 2014, Art. no. hal-00922537. [Online]. Available: https://hal.inria.fr/hal-00922537/document
[17] A. Ribes, J. Cerquides, Y. Demiris, and R. Lopez de Mántaras, "Active 939 learning of object and body models with time constraints on a humanoid 940 robot," IEEE Trans. Cogn. Develop. Syst., vol. 8, no. 1, pp. 26-41, 941 Mar. 2016, doi: 10.1109/TAMD.2015.2441375.

[18] J. Perkell et al., "The sensorimotor control of speech production," 943 in Proc. 1st Int. Symp. Meas. Anal. Model. Human Functions, 2001, 944 pp. 359-365.

[19] D. K. Oller and R. E. Eilers, "The role of audition in infant babbling," 946 Child Develop., vol. 59, no. 2, pp. 441-449, 1988.

[20] K. Ejiri, "Relationship between rhythmic behavior and canonical 948 babbling in infant vocal development," Phonetica, vol. 55, no. 4, 949 pp. 226-237, 1998.

[21] P.-Y. Oudeyer, F. Kaplan, and V. V. Hafner, "Intrinsic motivation systems 95 for autonomous mental development," IEEE Trans. Evol. Comput., 952 vol. 11, no. 2, pp. 265-286, Apr. 2007. seeking, curiosity, and attention: Computational and neural mecha- 955 nisms," Trends Cogn. Sci., vol. 17, no. 11, pp. 585-593, 2013.

[23] B. Galantucci, C. A. Fowler, and M. T. Turvey, "The motor theory of 957 speech perception reviewed," Psychonomic Bull. Rev., vol. 13, no. 3, 958 pp. 361-377, 2006.

[24] J.-L. Schwartz, A. Basirat, L. Ménard, and M. Sato, "The perception- 960 for-action-control theory (PACT): A perceptuo-motor theory of speech 961 perception," J. Neurolinguist., vol. 25, no. 5, pp. 336-354, 2012.

[25] S. Tremblay, D. M. Shiller, and D. J. Ostry, "Somatosensory basis of 963 speech production," Nature, vol. 423, no. 6942, pp. 866-869, 2003.

[26] S. M. Nasir and D. J. Ostry, "Speech motor learning in profoundly deaf 965 adults," Nature Neurosci., vol. 11, no. 10, pp. 1217-1222, 2008.

[27] S. N. Iyer and D. K. Oller, "Prelinguistic vocal development in infants 967 with typical hearing and infants with severe-to-profound hearing loss," 968 Volta Rev., vol. 108, no. 2, pp. 115-138, 2008.

[28] T. Ito, M. Tiede, and D. J. Ostry, "Somatosensory function in speech 970 perception," Proc. Nat. Acad. Sci. USA, vol. 106, no. 4, pp. 1245-1248, 971 2009.

[29] C. Moulin-Frier and P.-Y. Oudeyer, "The role of intrinsic motivations in 973 learning sensorimotor vocal mappings: A developmental robotics study," 974 in Proc. Interspeech, Lyon, France, 2013, pp. 1268-1272.

[30] S. Calinon, Robot Programming by Demonstration. Lausanne, 976 Switzerland: EPFL Press, 2009.

[31] D. W. Scott, Multivariate Density Estimation: Theory, Practice, and 978 Visualization. Hoboken, NJ, USA: Wiley, 2015.

Juan Manuel Acevedo-Valle, photograph and biography not available at the 980 time of publication.

Cecilio Angulo, photograph and biography not available at the time of 982 publication.

Clement Moulin-Frier, photograph and biography not available at the time 984 of publication. 


\title{
Autonomous Discovery of Motor Constraints in an Intrinsically Motivated Vocal Learner
}

\author{
Juan Manuel Acevedo-Valle, Cecilio Angulo, and Clement Moulin-Frier
}

\begin{abstract}
This paper introduces new results on the modeling of 2 early vocal development using artificial intelligent cognitive archi3 tectures and a simulated vocal tract. The problem is addressed 4 using intrinsically motivated learning algorithms for autonomous 5 sensorimotor exploration, a kind of algorithm belonging to the 6 active learning architectures family. The artificial agent is able 7 to autonomously select goals to explore its own sensorimotor \& system in regions, where its competence to execute intended goals is improved. We propose to include a somatosensory system to provide a proprioceptive feedback signal to reinforce learn11 ing through the autonomous discovery of motor constraints. ${ }_{12}$ Constraints are represented by a somatosensory model which 13 is unknown beforehand to the learner. Both the sensorimotor 14 and somatosensory system are modeled using Gaussian mixture 15 models. We argue that using an architecture which includes a 16 somatosensory model would reduce redundancy in the sensori17 motor model and drive the learning process more efficiently than 18 algorithms taking into account only auditory feedback. The role 19 of this proposed system is to predict whether an undesired colli20 sion within the vocal tract under a certain motor configuration 21 is likely to occur. Thus, compromised motor configurations are 22 rejected, guaranteeing that the agent is less prone to violate its 23 own constraints.
\end{abstract}

Index Terms-Active learning, early vocal development, 25 Gaussian mixture models (GMMs), intrinsic motivations, sen26 Sorimotor exploration.

27

28

${ }_{20} \mathbf{1}$ $\mathrm{N}$ RECENT years, there has been an increasing interest in using robots to perform daily life activities in the so presence of humans. As robot-human interactions become ${ }_{31}$ common then human-like communication systems become 32 more relevant to robotics. Speech is one of the most studied зз communication systems because it allows human-spoken lan34 guage. However, as mentioned in [1], the idea that speech is a 35 deeply encrypted "code" prevails among the speech specialists

Manuscript received July 19, 2016; revised November 18, 2016 and February 20, 2017; accepted April 14, 2017. This work was supported by the PATRICIA Research Project through the Spanish Ministry of Economy and Competitiveness under Grant TIN2012-38416-C03-01. The work of J. M. Acevedo-Valle was supported by CONACYT under Grant 216872 . (Corresponding author: Juan Manuel Acevedo-Valle.)

J. M. Acevedo-Valle and C. Angulo are with the GREC Research Group, Universitat Politècnica de Catalunya, 08028 Barcelona, Spain (e-mail: juan.manuel.acevedo.valle@upc.edu).

C. Moulin-Frier was with Flowers team, Inria/ENSTA-Paristech, 33405 Bordeaux, France. He is now with the SPECS Laboratory, Universitat Pompeu Fabra, 08018 Barcelona, Spain.

Color versions of one or more of the figures in this paper are available online at http://ieeexplore.ieee.org.

Digital Object Identifier 10.1109/TCDS.2017.2699578 and cracking this code is still an unsolved problem. Some of 36 the mysteries about speech might be solved if we are able to understand all the mechanisms underlying early speech acquisition in children. Thus, this paper, provides new results to contribute to the study of early speech development using machines.

Developmental robotics is a relatively novel approach, it 42 aims at understanding and modeling the role of developmental ${ }_{43}$ processes in the emergence of complex behaviors, including 44 social ones. Its goal is twofold, on the one hand it is used to 45 build more efficient cognitive machines applying developmen- 46 tal theories, and on the other hand it also provides insights into $\quad 47$ human developmental mechanisms, especially during infancy. 48 A deeper understanding of these mechanisms would explain 49 how human beings develop from infancy to functional adults 50 capable of solving highly complex cognitive tasks [2].

Autonomous robot design could notably benefit from 52 the available knowledge of biological science and self- 53 organization theories [3]. Deep understanding of the embod- 54 iment paradigm is paramount to integrate that knowledge 55 into robotics. This paradigm is also well represented by 56 the quote "understanding by building" [4]. It states that the ${ }_{57}$ behavior of an agent is not only the result of a system 58 control structure, but also a result of complex interactions 59 with its ecological niche, its morphology, and its material 6 properties [3], [4].

In this paper, language emergence is studied according 62 to behavioral and neurophysiological evidence, moreover the role of motor constraints is especially considered. The main assumption is that early vocal development can be studied as a result of embodiment, self-organization, and emergence 66 mechanisms produced by human evolution. In general, studies ${ }_{67}$ have shown that infants show preparedness to acquire natural 68 language. Motor, perceptual, social, and learning ability con- 69 straints, and their maturation during infant development play 70 a key role in the emergence of language [1].

Equally important, machine learning techniques have 72 rapidly evolved, providing developmental robotics with 73 interesting approaches as active learning. In contrast to the 74 more usual passive learning algorithms, active learning data 75 are collected in order to minimize a given property of the 76 learning process, e.g., the uncertainty [5] or the prediction 77 error [6] of a model. This family of algorithms is of partic- 78 ular interest for developmental robotics. During sensorimotor 79 exploration they allow the agent to focus on parts of the sen- 80 sorimotor space in which exploration is expected to improve 81 the quality of the learned model [7]. 
83 The contribution of this paper is extending the study 84 of early language development using intrinsically motivated 85 exploration algorithms. Herein, we provide new simulation 86 results showing the suitability of these algorithms in the self87 exploration of sensorimotor vocal spaces. The theoretical basis 88 of the probabilistic models used to represent knowledge is also 89 provided. Furthermore, we propose an architecture that could 90 be used to study the role of constraints during sensorimotor 91 exploration in embodied agents. Finally, it is worth mentioning 92 that the learning algorithm presented herein could be applied 93 to any system subjected to constraints in order to improve 94 learning progress.

95 The remainder of this paper is organized as follows. 96 Section II introduces related works. Section III highlights 97 the role of intrinsic motivations and proprioceptive feedback 98 in vocal development. The experiment setup is described 99 in Section IV and results are presented and discussed in 100 Section V. Finally, the conclusions are presented in Section VI.

\section{1 \\ II. RELATED WORK}

102 This paper revisits and expands the investigation introduced 103 in [8] and [9]. In [9], an intrinsically motivated exploration 104 architecture was proposed for the study of the developmental 105 stages emergent during the early vocal development of infants. 106 For the experimentation the simulated ear-vocal tract model 107 DIVA [10] was used. In spite of the relevance of its results, 108 the motor constraints and the somatosensory system were 109 neglected in [9]. However, morphological constraints play a 110 key role in speech acquisition. Therefore, a new exploration 111 algorithm proposed in [8] to incorporate motor constraints 112 awareness using a somatosensory model. In the past, some 113 studies have tried to explain the emergence of developmental 114 stages during the vocal development, assuming their exis115 tence, but those stages were bridged using hard-coding for 116 experimentation [10]-[13].

117 In [14], an approach for inverse kinematics learning in 118 redundant systems was presented. It was demonstrated that 119 goal babbling can be advantageous in learning in the early 120 stages of development, as observed in developmental theo121 ries. In parallel, [15] presented an intrinsically motivated goal 122 exploration approach for the active learning of inverse mod123 els. This approach was applied to the vocal sensorimotor space 124 exploration in [9] and [16]. The algorithm considered in this 125 paper extends intrinsically motivated exploration in the goal 126 space to include motor constraints. Considering both motor 127 and perceptual constraints during learning and exploration is 128 crucial to design cognitive architectures for motor control.

129 Among the efforts to model the acquisition of speech there 130 is the DIVA model [10]. It aims to imitate the underlying 131 neurophysiological mechanisms for speech acquisition and 132 production. The cognitive architecture of the system is an ${ }_{133}$ artificial neural network. The model includes the premotor, 134 motor, auditory and somatosensory cortical areas, and simu135 lated ear-vocal tract system. In [10], the somatosensory model 136 was effectively integrated into the acquisition and production 137 of speech processes. It was not used as an element to integrate 138 motor constraints but as an extra source of sensory-feedback.
The ear-vocal tract component of the DIVA model is used in 139 this paper, as it was in [8] and [9].

Finally, another interesting contribution was the active learn- 141 ing architecture presented in [17] which considered time con- 142 straints. This paper proposed a music performance imitation 143 scenario and implemented a learning architecture able to learn 144 a musical instrument model and a body capabilities model; the 145 architecture is also able to imitate a sequence of sound, while ${ }_{146}$ simultaneously kinematic errors, due to the control architec- 147 ture, are corrected. Similar to [9], models employed in [17] 148 were based on Gaussian mixture models (GMMs).

\section{Early Vocal Development in Machines}

Human speech production is one of the most complex motor 151 acts performed by any living being [18]. Producing a linguis- 152 tic message that can be understood by another human requires 153 coordinating many degrees of freedom in the respiratory, 154 laryngeal, and supraglottal articulatory system.

155

How infants acquire the complex ability to control speech 156 production and in general how they learn language remains a 157 matter of research [1]. It has been pointed out that strong 158 regularities can be observed in the structure of the vocal 159 development process independently of interindividual differ- 160 ences [1], [19]. In general, the infant first discovers how to 161 control phonation, then focuses on vocal variations of unartic- 162 ulated sounds and finally automatically discovers and focuses 163 on babbling with articulated proto-syllables. In [18], some 164 experiments suggested that goals of speech movements are 165 auditory in nature and maintenance of motor command maps 166 to auditory results is performed with auditory feedback. $\quad{ }_{167}$

It is important to inquire into the developmental assump- 168 tions considered in the experiments in [8] and [9], as this 169 paper is based on those experiments. Regarding the infant 170 development stages mentioned in [1], our experiments con- 171 sider the developmental stage known as canonical babbling 172 (CB) [20] and the beginning of language-specific speech pro- 173 duction [1]. Results suggest that during CB infants learn to 174 control their ear-vocal tract system based on auditory feed- 175 back. Nevertheless, when infants begin to babble they do it 176 regardless of the audibility of their vocalizations. CB could 177 be the result of a natural tendency of infants to move their 178 body parts rhythmically motivated by sensory feedback [20]. 179

Consistent with the theory, we assumed a simplified expla- 180 nation that the artificial agent is exploring its ear-vocal tract 181 system choosing auditory goals and evaluating the result. 182 Therefore, our cognitive architecture allows the agent to 183 explore regions, where the competence to produce intended 184 sounds is improved. However, we also endow the agent with 185 autonomous mechanisms to discover constraints in order to 186 drive the exploration. To accomplish that objective, previously ${ }_{187}$ proposed active learning architectures and the proprioceptive 188 feedback concept are combined.

\section{A. Intrinsically Motivated Exploration Architectures}

Among the vast number of active learning architectures, 191 this paper considers the exploration architecture proposed 192 
193 by [15]. This architecture reproduces the formalism of intrin194 sic motivation inspired by psychological literature as proposed 195 previously in [21] and [22]. Using goal babbling, intrinsi196 cally motivated exploration aims to minimize the error of an 197 agent to reach self-generated goals measured according to a 198 competence function. This architecture allows artificial agents 199 to efficiently and actively explore and generate maps from 200 motor capacities to perceived results. Therefore, exploration 201 occurs over regions in which agents perceive they are becom202 ing more competent to reach self-generated goals. Intrinsically 203 motivated exploration architectures were originally designed 204 to actively learn inverse models of high-dimensional input205 output spaces. This architecture was later extended by [9] to 206 study self-organization in early vocal development stages in 207 infants and robots.

208 Intrinsically motivated learning algorithms have shown 209 favorable results in previous experiments to learn sensorimotor 210 coordination skills in redundant nonlinear high-dimensional 211 mappings which share many mathematical properties with 212 vocal spaces. Moulin-Frier et al. [9] used a simulated ear213 vocal tract system to study the emergence of developmental 214 stages implementing intrinsically motivated exploration. They 215 argued that the development of the agent self-organizes into 216 vocal developmental sequences. The results presented therein 217 opened the door to a new approach in vocal development 218 to be explored. This paper introduces a methodology which 219 enhances intrinsically motivated architectures with constraint 220 awareness.

\section{B. Proprioceptive Feedback}

222 Some of the most adopted theories of speech state that 223 speech production is organized in terms of motor control sig224 nals and their associated vocal tract configurations which has 225 been corroborated by several experimental results [23], [24]. 226 Nevertheless, we adopt the simplified hypothesis that speech 227 goals are defined acoustically and maintained by auditory 228 feedback [18]. CB is a rhythmic behavior that, with some dif229 ferences, emerges in both, normally developing infants and 230 infants with hearing loss. When infants start to babble, they ${ }_{231}$ do it regardless of the audibility result (i.e., they produce audi232 ble and voiceless vocalizations). However, evidence suggests 233 that, around the onset of $\mathrm{CB}$, infants learn to vocalize based 234 on auditory feedback [1], [20].

${ }_{235}$ How the somatosensory system ${ }^{1}$ affects the ear-vocal tract 236 exploration is an open question that was not previously ${ }_{237}$ approached in [9]. However, the relevance of the somatosen238 sory system for speech has been shown in different exper239 iments, for instance the results in deaf individuals suggest 240 that somatosensory inputs related to movement play a more 241 important role in speech production than what was thought 242 before [25], [26]. Furthermore, the fact that CB also emerges 243 in deaf infants suggests that somatosensory feedback must play 244 a more relevant role during the prelinguistic vocal development 245 in infants [27].

\footnotetext{
${ }^{1}$ Strictly, the somatosensory system is also a sensorimotor system. In future works, we will distinguish two sensorimotor systems: 1) the auditory-motor system and 2) the somatosensory-motor system.
}

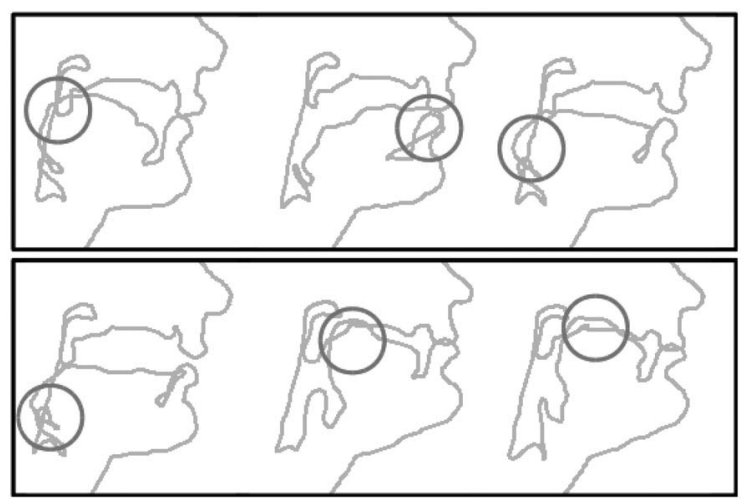

Fig. 1. Examples of articulatory configurations that produce collisions in the DIVA vocal tract model.

In [28], a robotic device able to generate patterns of facial 246 skin deformation related to certain speech productions was 247 used. The results showed that when the facial skin is stretched 248 whilst subjects are listening to words, the sounds they hear are 249 altered. Thus, theory and results suggest that the somatosen- 250 sory system is involved in speech perception. Following 251 this hypothesis, improvements can made to the experiments 252 proposed in [9] by including a somatosensory system to endow 253 the learner with physical constraint awareness. 254

In [8], the foundations of a simplified architecture were 255 established allowing us to include physical constraints to the 256 learning process through a proprioceptive signal, similar to ${ }_{257}$ the ability to feel pain in humans. The open source DIVA ${ }_{258}$ model $^{2}[10]$ provides a synthesizer that represents the human 259 vocal tract and ear systems. The DIVA model also includes 260 a somatosensory system, but in spite of it, there is a lack of 261 physical constraints in the DIVA vocal tract. The absence of 262 constraints allows the execution of motor commands that lead 263 to collisions or articulatory superpositions. Both circumstances 264 lead to no phonation and moreover, the latter is a contradictory 265 result since it lacks physical sense, as shown in Fig. 1.266

To overcome the drawbacks caused by the lack of con- 267 straints, we introduce a somatosensory system. This new 268 element, not considered in [9], is based on an area function 269 which is a vector descriptor of the vocal tract shape. It con- 270 sists of a mechanism that evaluates if an exploratory motor 271 command produces a collision or superposition of articulatory 272 tissues, the system generates a proprioceptive signal. Using the 273 data generated with this mechanism, the agent builds a map 274 from motor commands to proprioceptive results. This map is 275 used to predict which motor commands may lead to undesired 276 collisions, so they may be rejected, forcing the agent to choose 277 a new auditory goal. In the next section, this mechanism is 278 explained in detail.

\section{Proposed Architecture}

280

The experimental architecture proposed in this paper to 281 study the early vocal development in machines is shown 282 in Fig. 2, where five elements interact. These elements are 283

\footnotetext{
${ }^{2} \mathrm{http}: / /$ www.bu.edu/speechlab/software/diva-source-code/
} 


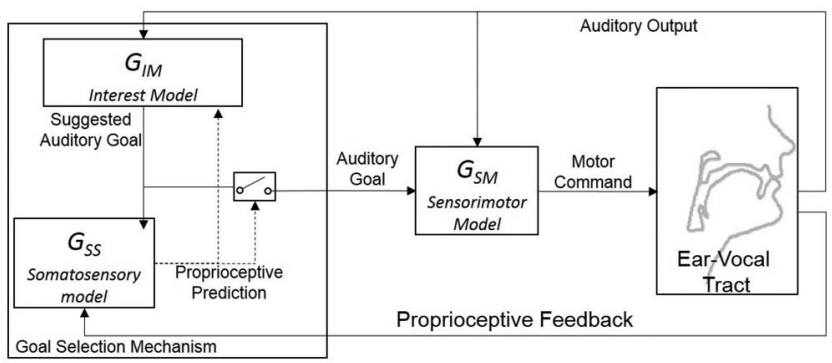

Fig. 2. Experimental architecture. It is composed by five interacting modules, two of them contained within the ear-vocal tract module (the sensorimotor system and the somatosensory system).

284 introduced below and explained in detail in the coming 285 sections.

\section{A. Sensorimotor System}

${ }_{310}$ The DIVA vocal tract configuration is determined by the 311 position of ten articulators and three phonation parameters. ${ }_{312}$ Along this paper, only seven articulators and two phonation 313 parameters (voicing and glottal pressure) are considered [9]. 314 Articulators and voicing parameter motor dynamics are mod315 eled as overdamped second order systems

$$
\ddot{x}+2 \zeta \omega_{0} \dot{x}+\omega_{0}^{2}(x-m)=0
$$

317 with $\zeta=1.01$ and $\omega=(2 \pi / 0.8)$ representing the damping 318 factor and the natural frequency, respectively. The duration of 319 each vocal experiment in seconds is 0.8 , whereas $m$ and $x$ rep320 resents the desired articulator position (motor command) and 321 the current articulator position, respectively. During each vocal 322 experiment two different motor commands are introduced for 323 each of the seven articulators and the two voicing parameters: 324 one for the $0-250 \mathrm{~ms}$ window and another for the remain325 ing time. Thus, each motor command is represented by an

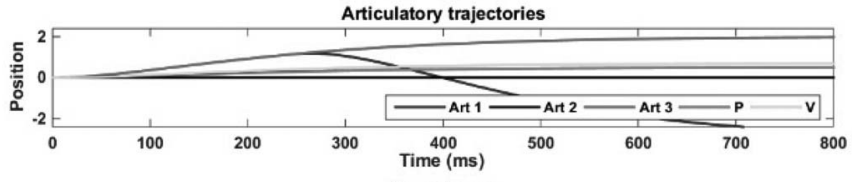

Sound wave
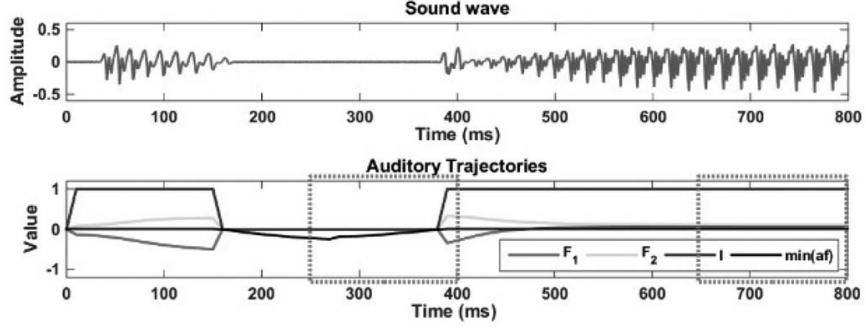

Fig. 3. Vocalization experiment structure. The upper plot shows the articulatory trajectories, from 0 to $250 \mathrm{~ms}$, the commands for Art1, Art2, and Art3 are set to 2, 0 , and 2, respectively, whereas the glottal pressure and voicing are both set to 0.5 . From 250 to $800 \mathrm{~ms}$, the commands for Art1, Art2, and Art3 are set to $-3,0$, and 2, respectively, whereas the glottal pressure and voicing are both set to 0.7 . The remaining motor commands are set to zero. The middle plot represents the speech sound wave signal. The bottom plot shows the auditory trajectories. The dotted outlined boxes represent the perception time windows from 250 to $400 \mathrm{~ms}$ and the second from 650 to $800 \mathrm{~ms}$. The auditory output $s$ are determined from the average of each trajectories along each one of the time windows. Whereas the proprioceptive feedback $p$ is determined by the average value of $\min \left(a_{f}\right)$.

18-D vector. The auditory output of a human vocalization can 326 be described by its formant frequencies. We consider the first ${ }_{327}$ two formant frequencies, $F_{1}$ and $F_{2}$, along with an intonation 328 signal $I$. The intonation signal is 1 when phonation occurs ${ }_{329}$ and 0 otherwise, two conditions are required for phonation 330 to occur: 1) the area function $a_{f}$ of the vocal tract must be ${ }_{331}$ positive elsewhere and 2) the voicing and pressure parameters ${ }_{332}$ must be positive. The area function is a vector function that ${ }_{33}$ describes the transversal shape of the vocal tract. 334

During the vocalization the auditory output of the system 335 is observed along two time windows, the first from 250 to 336 $400 \mathrm{~ms}$ and the second from 650 to $800 \mathrm{~ms}$. The value of each ${ }_{337}$ auditory output is averaged for each time window, the result 338 is a 6-D output signal (two formants and the intonation, hence ${ }_{339}$ three values, per each of the two time windows). In Fig. 3, 340 we reproduce the vocalization representation shown in [9]. To ${ }_{341}$ be consistent with the co-articulated nature of speech, only ${ }_{342}$ two perceptual windows are used [1]. However, since only two ${ }_{343}$ portions of the vocalization are considered, a lot of information 344 is lost. For instance, it is shown in [23] the continuum of co- 345 articulated gestures. Therefore, future works should consider 346 studying the continuum of speech gestures and self-structuring 347 of vocalizations.

\section{B. Somatosensory System}

349

In Fig. 3, it is shown that the area function $a_{f}$ is observed ${ }_{350}$ during both perception time windows. The minimal value of 351 the area function $\min \left(a_{f}\right)$ would be zero when the vocal tract 352 is closed at any point and negative values mean that some tis- ${ }_{353}$ sues are overlapped, which does not have physical meaning. 354 However, in some cases it might be interpreted as the tongue 355 being bitten. In other cases it might represent high pressure 356 between the tongue and the palate, which might be interesting ${ }_{357}$ 
358 to the learner in a realistic scenario, where motor constraints 359 are not violated. In general, we made a strong assumption that 360 any motor constraint violation over a threshold is uncomfort361 able or painful. Hence, the average value of $\min \left(a_{f}\right)$ in each 362 perception time window is used to generate a proprioceptive 363 feedback signal $p$ : if the average of $\min \left(a_{f}\right)$ is lower than a 364 threshold for any perception window, then the configuration 365 is evaluated as a undesired collision with $p=1$, and $p=0$ 366 otherwise.

\section{${ }_{367}$ C. Sensorimotor Model}

368 GMMs are linear combinations of multivariate Gaussian 369 distributions that represent clusters of data. They have 370 been previously used to represent nonlinear redundant 371 maps [17], [21], [29] in order to solve the inverse problem 372 of inferring input motor commands from desired sensory out373 puts. GMMs can be learned using an online variant of the 374 expectation-maximization (EM) algorithm in order to learn 375 incrementally from incoming data [30]. Here, the algorithms 376 used to train GMMs are based on the open source tools ${ }^{3}$ 377 associated with [30], and modified according to our problem 378 requirements. The three models in the experimental setup are 379 probabilistic representations in the form of GMMs, obtained 380 using data collected from experiments with the DIVA ear-vocal 381 tract. A detailed explanation of the GMMs training is provided 382 below.

383 We assume that an $n$-dimensional input command space ${ }_{384} X \in \mathbb{R}^{n}$ is mapped to an $m$-dimensional output space $Y \in \mathbb{R}^{m}$, 385 through a transform function $y=f(x)+\varepsilon$, where $y \in Y$, 386 $x \in X$ and $\varepsilon$ is random noise. When a dataset of couples ${ }_{387}(x, y)$ is available, the EM-algorithm is used to obtain a GMM 388 which is defined by the parameters $\left\{\pi_{j}, \mu_{j}, \Sigma_{j}\right\}_{j=1}^{K}$, where $\pi_{j}$, $389 \mu_{j}$, and $\Sigma_{j}$ are, respectively, the prior probability, the distribu390 tion centroid and the covariance matrix of the $j$ th Gaussian, for ${ }_{391} j=1,2, \ldots, K$, being $K$ the number of Gaussian components. 392 From [30], Gaussian mixture regression (GMR) is applied to 393 compute the conditional probability distribution $P(X \mid y)$ in the 394 input space $X$ given a desired output $y$. Once it is computed, 395 the value $x^{*} \in X$ is selected such that it maximizes $P(X \mid y)$.

396 To obtain the input $x$ that maximizes the probability to pro397 duce the output $y$, the GMR process first defines the partitioned 398 vector $z \in X \times Y$, where

399

$$
z=\left(\begin{array}{l}
x \\
y
\end{array}\right)
$$

400 For each Gaussian $j$ in the GMM the partitions

$$
401 \quad \mu_{j}=\left(\begin{array}{l}
\mu_{j}^{x} \\
\mu_{j}^{y}
\end{array}\right) \quad \text { and } \quad \Sigma_{j}=\left(\begin{array}{cc}
\Sigma_{j}^{x} & \Sigma_{j}^{x y} \\
\Sigma^{y x} & \Sigma_{j}^{y}
\end{array}\right)
$$

402 are considered to compute the conditional probability distribu${ }_{403}$ tion $P_{j}(X \mid y) \sim N_{j}\left(\hat{\mu}_{j}, \hat{\Sigma}_{j}\right)$ in the input space $X$ given a desired 404 output $y$, where

$405 \hat{\mu}_{j}=\mu_{j}^{y}+\Sigma_{j}^{y x}\left(\Sigma_{j}^{x}\right)^{-1}\left(x-\mu_{j}^{x}\right), \hat{\Sigma}_{j}=\Sigma_{j}^{y}+\Sigma_{j}^{y x}\left(\Sigma_{j}^{x}\right)^{-1} \Sigma_{j}^{x y}$.

406

\footnotetext{
${ }^{3} \mathrm{http}: / /$ www.calinon.ch/sourcecodes.php
}

Considering that $P(X \mid y)$ is at its maximum when $x=\hat{x}_{j}=\hat{\mu}_{j}, 407$ then a natural selection for $x$ in order to produce $y$ is $\hat{x}_{j}$. But 408 we have $K$ candidates for $x$, hence it is necessary to compute 409

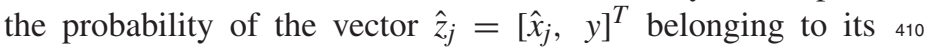
generator Gaussian as

$$
P\left(\hat{z}_{j}\right)=\pi_{j} \frac{1}{\sqrt{(2 \pi)^{K}\left|\Sigma_{j}\right|}} e^{-\frac{1}{2}\left(\left(\hat{z}_{j}-\mu_{j}\right)^{T} \Sigma_{j}^{-1}\left(\hat{z}_{j}-\mu_{j}\right)\right)}
$$

and finally the point $z^{*}=\hat{z}_{j}$ that maximizes $P\left(\hat{z}_{j}\right)$ is selected as 413 the point that better fits the model. In other words, according 414 to our prior knowledge of $f(x), z^{*} \in f(x)$, we infer that the 415 output $y$ is generated by $\hat{x}_{j}$. 416

Taking into account the above for the sensorimotor model, 417 an 18-D motor command space $M$, with $m \in M$, is defined 418 for the vocal tract articulatory configuration. A 6-D auditory 419 output space $S$, with $s \in S$, is also defined, the agent being able ${ }_{420}$ to observe $s$ according to $s=f(m)+\sigma$, where $\sigma \sim N(0,0.01) \quad 421$ is Gaussian noise. The aim is to find a GMM that solves the 422 inverse problem $m=f^{-1}\left(s_{g}\right)$, where $s_{g}$ is an auditory goal. ${ }_{423}$

We define a GMM, $G_{\mathrm{SM}}$, to model the sensorimotor system, 424 with $X=M$ and $Y=S$. Such a model allows computation 425 of the inverse model $P\left(M \mid s_{g}\right)$ using GMR. At the beginning 426 of the experiment, $m$ is selected either, randomly or accord- 427 ing to the interest for initializing the inverse sensorimotor 428 model $m \sim f^{-1}\left(s_{g}\right) \sim P\left(M \mid s_{g}\right)$ around a specific region of ${ }_{429}$ the sensorimotor space. After the initialization stage, the agent 430 starts to select new auditory goals, according to the interest 431 model explained below. In order to reduce memory storage 432 requirements, we consider a generative method for the train- 433 ing stage, which means that the model is trained using the last 434 $N_{\text {SM }}$ samples obtained from experimentation along with $\quad 435$

$$
N_{\text {old }}=\left\lceil\frac{(1-\alpha) N_{\mathrm{SM}}}{\alpha}\right\rceil \text { samples }
$$

generated using $G_{\mathrm{SM}}$, where $\alpha \in[0,1]$ is the forgetting rate. ${ }_{437}$

\section{Interest Model for Auditory Goals}

The interest model for auditory goals endows the learner the 439 ability to select goals that maximize the expected competence 440 progress in order to improve the quality of its sensorimotor 441 model, resulting in better control over it. It is derived from 442 the model proposed in [9]. The competence value for a goal 443 is defined by

$$
c=e^{-\left|s_{g}-s\right|}
$$

where $s_{g}$ is the auditory goal and $s$ is the actual auditory pro- 446 duction after executing a motor command $m \sim P\left(M \mid s_{g}\right)$. To 447 construct the interest model, the auditory goal space is aug- 448 mented with two extra dimensions: 1) the competence $c \in C \quad{ }_{449}$ and 2) time tag $t \in T$. The number of vocalizations $N_{\text {IM }}$ con- 450 sidered to build the interest model is fixed. A GMM, $G_{\mathrm{IM}}$, with 451 $K_{\text {IM }}$ components will be computed from the 8-D data set with 452 $N_{\text {IM }}$ samples of the augmented goal space. To initialize this 453 model, some auditory results from the initialization of $G_{\mathrm{SM}} 454$ are selected as the first auditory goals $s_{g}$.

455

Those Gaussian components in $G_{\mathrm{IM}}$ that, according to the 456 covariance matrices $\Sigma_{j}$, contain goals that will likely increase 457 
458 the competence progressively are considered to build a prob459 abilistic distribution $P(S)$ over the auditory space. In order to 460 build $P(S)$, the components in $G_{\mathrm{IM}}$ are weighted according to 461 their time-competence covariance magnitudes. Thus, $P(S)$ will 462 prioritize goals in regions, where competence is expected to 463 increase. Finally, a sample $s_{g}$ is drawn from $P(S)$ for the next 464 vocalization experiment. Model training is performed every 465 time the agent has performed $n_{\mathrm{IM}}$ experiments, using the last $466 N_{\text {IM }}$ vocalizations.

\section{${ }_{467}$ E. Somatosensory Model}

${ }_{468}$ For the somatosensory model we consider the 18-D motor 469 command space $M$, with $m \in M$, and a new binary propriocep470 tive output space $P=\{0,1\}$, with $p \in P$. If a vocal production 471 leads to undesired contacts, then $p=1$, otherwise $p=0$. A 472 map $g$ is assumed to exist such that $p=g(m)$ and the agent 473 can observe $p$ for each vocal experiment. Thus, it is possible 474 to find a GMM $G_{\mathrm{SS}}$, with $X=M$ and $Y=P$, that allows 475 computation of the probability distribution $P(P \mid m)$ applying ${ }_{476}$ GMR, and determine when a motor command $m$ is likely to 477 lead to an undesired collision in the vocal tract.

478 The inverse sensorimotor model $G_{\mathrm{SM}}$ and the somatosensory 479 model $G_{\mathrm{SS}}$ are initialized together. When an auditory goal $s_{g}$ 480 has been selected, $m$ is computed using $P\left(M \mid s_{g}\right)$. Next, to 481 predict the value of $p, P(P \mid m)$ is used. If the prediction sug${ }_{482}$ gests that $m$ will produce $p=1$ then $s_{g}$ is rejected, otherwise ${ }_{483} s_{g}$ and $m$ are accepted. If $s_{g}$ is rejected, then $G_{\mathrm{IM}}(S)$ is recom${ }_{484}$ puted without considering the Gaussian component in $G_{\mathrm{IM}}$ that 485 generated $s_{g}$, this mechanism decreases the prior of the con${ }_{486}$ flicting Gaussian in $G_{\mathrm{IM}}$. The new $G_{\mathrm{IM}}(S)$ is used to select a ${ }_{487}$ new goal $s_{g}$, and the process is repeated until $s_{g}$ is accepted. ${ }_{488}$ During the agent's life, the model $G_{S S}$ is trained when $G_{S M}$ is 489 trained using the previously described generative mechanism.

\section{F. Self-Exploration Algorithm}

491 The self-exploration architecture with motor constraints 492 self-awareness, first proposed in [8] for ear-vocal tract explo493 ration, is an extended version of [9]. The algorithm associated 494 with the cognitive architecture is shown in Algorithm 1. Our 495 extended self-exploration algorithm with goal babbling and 496 motor constraints self-awareness starts with the learner having 497 no experience in vocalizing. Models $G_{S M}$ and $G_{S S}$ are initial498 ized using random vocalizations with small values around the 499 neutral position of the articulators. The neutral position of the 500 pressure and voicing parameters are set to -0.25 to produce 501 no phonation, whereas for the articulators it is considered 0 , 502 i.e., the rest position. Model $G_{\mathrm{IM}}$ is also initialized.

${ }_{503}$ Then, in line 6 of Algorithm 1 the vocal learner agent selects 504 a goal $s_{g}$ for the next experiment according to the probabilis505 tic distribution $P(S)$ and the motor command $m$ is obtained 506 using the inverse model $G_{\mathrm{SM}}$ in line 7. The main feature of 507 this algorithm, different from similar architectures, is that in ${ }_{508}$ line $8 P(P \mid m)$ provides a prediction for $p$ that indicates if 509 the selected motor command is likely to produce an undesired 510 collision. From line 9 to 11 , if $p \approx 1$, the goal is rejected 511 and the probabilistic distribution $P(S)$ is updated, ignoring the

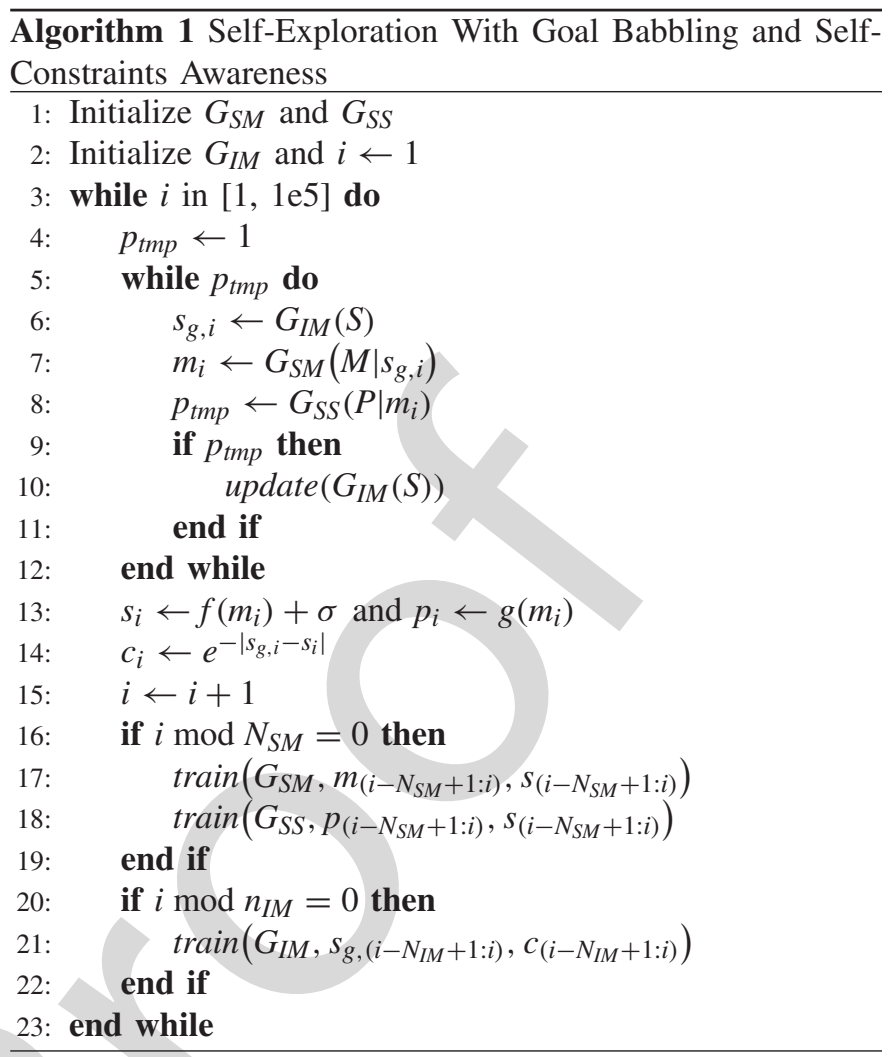

Gaussian component in $G_{\mathrm{IM}}$ that generated $s_{g}$ and the algo- 512 rithm goes back to line 6 . Otherwise, $p \approx 0$, both, $s_{g}$ and $m$ are ${ }_{513}$ accepted. Next, the motor command is executed with the vocal 514 tract and the agent observes $s$ and $p$ in line 13. In line 14, the 515 learner evaluates the competence value $c$. It also checks if we 516 are at the end of a learning episode, so models $G_{\mathrm{SM}}, G_{\mathrm{SS}}$, and ${ }_{517}$ $G_{\mathrm{IM}}$ are updated in lines 17,18 , and 21 , respectively. To pro- 518 vide objective evaluation elements, some experiments without 519 considering the somatosensory model for choosing goals are 520 also presented. In this later case, $s_{g}$ is always accepted, thus 521 line 4 is substituted with $p_{\text {tmp }}=0$ in Algorithm 1 .

\section{EXPERIMENTAL RESULTS}

523

Eighteen independent simulations using Algorithm 1 were ${ }_{524}$ run. All simulations consisted of half a million of vocaliza- 525 tions, including an initial vocalization set of 1000 random 526 samples. Nine different random seeds were considered to ${ }_{527}$ generate the same number of motor command sets from a ${ }_{528}$ uniform distribution. The limits for those motor commands ${ }_{529}$ related to the vocal tract articulators were $[-1,1]$, whereas 530 for motor commands related to the phonation parameters were ${ }_{531}$ $[0,0.7]$. Each set was used twice to initialize simulations of ${ }_{532}$ Algorithm 1, first without using the somatosensory model and ${ }_{53}$ second with it.

Considering as a reference the parameters used for simula- 535 tions in [8] and [9], a few variations in their values were tested. 536 First, when values for $K_{\mathrm{SM}}$ or $K_{\mathrm{SS}}$ are increased the inference ${ }_{537}$ error decreases slightly but the computation time grows con- 538 siderably. On the other hand if these values are decreased, the ${ }_{539}$ inference error increases considerably. Second, if the training 540 


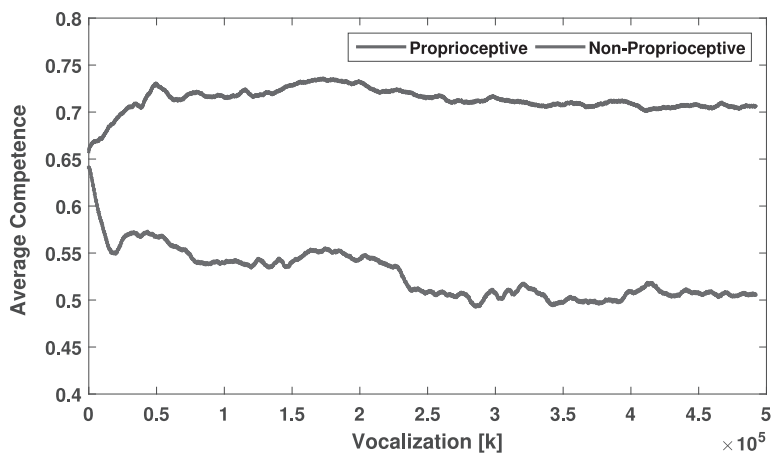

Fig. 4. Mean competence evolution during simulations using Algorithm 1 for nine different initialization data sets. Moving average of 5000 samples are considered to filter the results of each simulation. Results are shown in the case of proprioceptive and nonproprioceptive agents.

541 steps are increased for the somatosensory model and the senso542 rimotor model, then the training computational time increases 543 as $N_{\text {old }}$ in (6) increases proportionally, but no improvement 544 is obtained in the inference error. However, if these values 545 are decreased beyond the values used in [9], the mean infer546 ence error increases. Third, when $\alpha_{\mathrm{SM}}$ and $\alpha_{\mathrm{SS}}$ are larger than ${ }_{547} 0.1$ the competence progress is slower. Finally, the parame548 ters linked to the interest model allow a wider range of values 549 to be chosen obtaining similar results. For $K_{\mathrm{IM}}$ we observed 550 that values greater than or equal to 12 worked similarly, but 551 smaller values negatively impacted the competence progress. 552 Thus, the main parameters for all the simulations were kept 553 as in [8] and [9] as they performed better than other sim554 ulations in terms of exploration results and simulation time. 555 Summarizing, values were set to $K_{\mathrm{SM}}=28, N_{\mathrm{SM}}=400$, ${ }_{556} K_{\mathrm{SS}}=28, K_{\mathrm{IM}}=12, N_{\mathrm{IM}}=4800, n_{\mathrm{IM}}=12$, and the con557 tinuous sampling time used for the DIVA ear-vocal tract was ${ }_{558} t_{s}=10 \mathrm{~ms}$. The forgetting rate parameter $\alpha_{\mathrm{SM}}$ for $G_{\mathrm{SM}}$ starts 559 from 0.1 and decreases logarithmically to 0.05 after half a 560 million of vocalizations. On the other hand, $\alpha_{\mathrm{SS}}$ for $G_{\mathrm{SS}}$ was 561 chosen to be 0.05 through the whole simulation. ${ }^{4}$

${ }_{562}$ During the simulation, $G_{\mathrm{SM}}$ and $G_{\mathrm{SS}}$ are initialized as indi563 cated in line 1 of Algorithm 1 with the initial motor command 564 sets. Then, all the initial phonatory productions are used as 565 auditory goals to initialize the interest model $G_{\mathrm{IM}}$ as indicated 566 in line 2 of Algorithm 1. In this stage, $G_{S M}$ is used to infer the 567 motor commands that will likely produce the initial auditory 568 goals. These commands are executed without considering the 569 proprioceptive prediction $p$.

\section{A. On Competence and Contacts}

571 First of all, Fig. 4 represents the evolution of the compe572 tence parameter $c$ in (7) for self-generated auditory goals. To 573 obtain this plot, first the result of each simulation is filtered 574 using a 5000 samples moving average window. Next, simu575 lations are divided into two general groups: 1) proprioceptive 576 agents and 2) nonproprioceptive agents. Finally, the mean of

\footnotetext{
${ }^{4}$ Supplementary downloadable material provided by the authors is available at https://dx.doi.org/10.6084/m9.figshare.c.3676645.v1. After each experiment, 20 random samples from the last 1000 vocalizations were drawn to generate videos with audio. Videos of the experiments 1,5 , and 9 are provided.
}

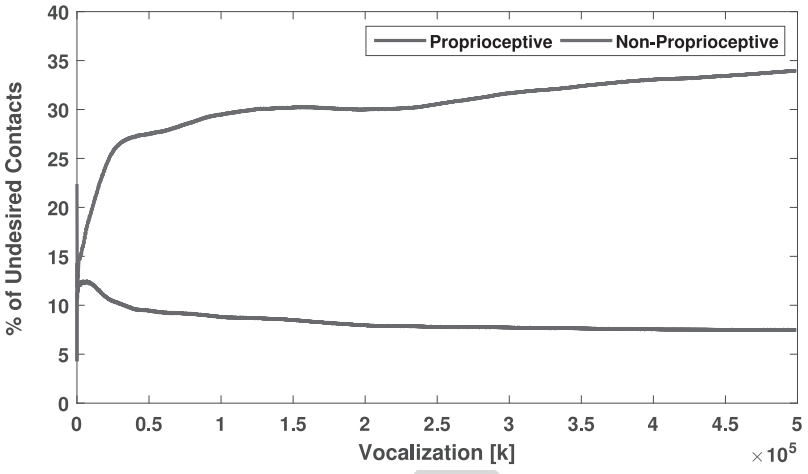

(a)

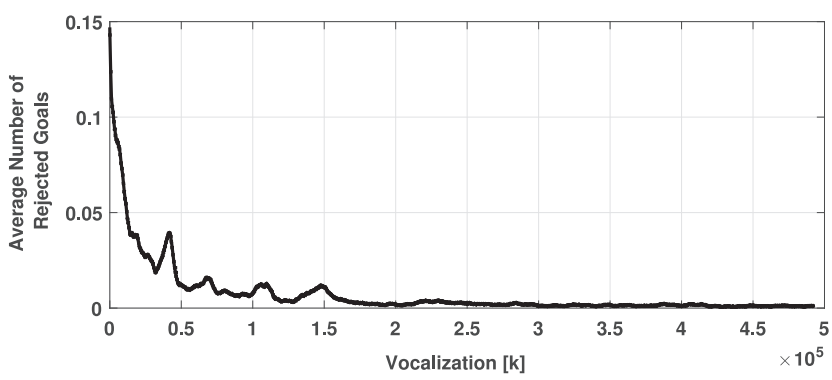

(b)

Fig. 5. Algorithm 1 simulation results. (a) Mean percentage of vocalizations producing undesired collisions considering all the simulated agents. Agents are grouped by proprioceptive and nonproprioceptive. The results of each agent are prefiltered considering a 5000 samples moving average. (b) Mean number of rejected goals using the proprioceptive prediction and considering all the simulated proprioceptive agents. The results of each agent are prefiltered considering a 5000 samples moving average.

TABLE I

Results CONSIDERING All DATA From Exploration

\begin{tabular}{|c|c|c|c|c|c|c|}
\hline \multirow{2}{*}{ Experiment } & \multicolumn{3}{|c|}{ Non-Proprioceptive } & \multicolumn{3}{|c|}{ Proprioceptive } \\
\cline { 2 - 7 } & Vol. & mean $(c)$ & \% Contacts & Vol & mean $(c)$ & $\%$ Contacts \\
\hline \hline 1 & 0.58 & 0.50 & $41.67 \%$ & 0.49 & 0.80 & $3.42 \%$ \\
\hline 2 & 0.47 & 0.50 & $37.50 \%$ & 0.49 & 0.76 & $3.88 \%$ \\
\hline 3 & 0.49 & 0.50 & $43.53 \%$ & 0.43 & 0.61 & $5.28 \%$ \\
\hline 4 & 0.47 & 0.54 & $30.83 \%$ & 0.52 & 0.73 & $9.03 \%$ \\
\hline 5 & 0.47 & 0.54 & $29.17 \%$ & 0.44 & 0.68 & $15.55 \%$ \\
\hline 6 & 0.56 & 0.59 & $23.01 \%$ & 0.52 & 0.70 & $8.18 \%$ \\
\hline 7 & 0.49 & 0.55 & $30.86 \%$ & 0.41 & 0.71 & $6.76 \%$ \\
\hline 8 & 0.57 & 0.54 & $35.49 \%$ & 0.43 & 0.68 & $8.57 \%$ \\
\hline 9 & 0.49 & 0.50 & $32.42 \%$ & 0.63 & 0.74 & $6.74 \%$ \\
\hline \hline Average & 0.51 & 0.53 & $33.83 \%$ & 0.48 & 0.71 & $7.49 \%$ \\
\hline Min & 0.47 & 0.50 & $23.01 \%$ & 0.41 & 0.61 & $3.42 \%$ \\
\hline Max & 0.58 & 0.59 & $43.53 \%$ & 0.63 & 0.80 & $15.55 \%$ \\
\hline
\end{tabular}

Note: Experiments with different vocalization initial sets for Proprioceptive and NonProprioceptive Agents. The volume of a the convex-hull described by the explored data, the mean value for the competence $c$, and the final percentage of contacts along the simulations are shown.

all the filtered results for each group is computed. The same 577 mechanism is considered to obtain the percentage of contacts 578 observed in Fig. 5(a).

Tables I and II show the volume of a convex hull cov- 580 ering the explored auditory region. They also display the 581 mean competence and the percentage of undesired contacts 582 at the end of the simulation. First, in Table I, descriptors are 583 computed considering all the vocalization during each simula- 584 tion. Second, in Table II, figures were computed considering 585 
TABLE II

RESUlTS WiTHOUT CONSIDERING VOCALIZATIONS WITH UNDESIRED CONTACTS

\begin{tabular}{|c||c|c|c|c|}
\hline \multirow{2}{*}{ Experiment } & \multicolumn{2}{c|}{ Non-Proprioceptive } & \multicolumn{2}{c|}{ Proprioceptive } \\
\cline { 2 - 5 } & Vol. & mean $(c)$ & Vol. & mean $(c)$ \\
\hline \hline 1 & 0.48 & 0.69 & 0.39 & 0.81 \\
\hline 2 & 0.38 & 0.67 & 0.39 & 0.78 \\
\hline 3 & 0.37 & 0.71 & 0.33 & 0.63 \\
\hline 4 & 0.40 & 0.65 & 0.42 & 0.77 \\
\hline 5 & 0.36 & 0.66 & 0.37 & 0.76 \\
\hline 6 & 0.44 & 0.67 & 0.42 & 0.74 \\
\hline 7 & 0.36 & 0.69 & 0.32 & 0.74 \\
\hline 8 & 0.45 & 0.67 & 0.35 & 0.71 \\
\hline 9 & 0.38 & 0.64 & 0.49 & 0.78 \\
\hline \hline Average & 0.40 & 0.67 & 0.39 & 0.75 \\
\hline Min & 0.36 & 0.64 & 0.32 & 0.63 \\
\hline Max & 0.48 & 0.71 & 0.49 & 0.81 \\
\hline
\end{tabular}

Note: Experiments with different vocalization initial sets for Proprioceptive and NonProprioceptive Agents. The volume of a the convex-hull described by the explored data and the mean value for the competence $c$ along the simulations are shown.

586 vocalizations without undesired collisions. Convex hulls pro${ }_{587}$ vide an insight regarding the size of the explored regions in 588 the auditory-space. They are computed considering formant 589 frequency dimensions $F_{11}, F_{21}, F_{12}$, and $F_{22}$.

590 In Fig. 4, results suggest that proprioceptive agents perform 591 better than those which are not endowed with proprioception. 592 We observe that at the beginning of the exploration the mean 593 average competence is very similar for both groups. However, 594 after the initialization the nonproprioceptive agents suffer an 595 important decrement of the competence, which also coincides 596 with a significant increment of the percentage of contacts in 597 Fig. 5(a). Table I also confirms the expected results accord598 ing to our hypothesis: using proprioceptive feedback drives 599 artificial agents to produce significantly fewer undesired con600 tacts and also increases the competence to reach self-generated 601 auditory goals.

602 Some observers might ask the reason of high competence 603 values at the beginning of the simulations. We argue that it is 604 an expected result as the competence computation begins when ${ }_{605} G_{\mathrm{IM}}$ is initialized with self-generated goals drawn from the ini606 tial auditory productions of the agent. In other words, $G_{\mathrm{SM}}$ and ${ }_{607} G_{S S}$ models are initialized around a set of initial vocalizations 608 and auditory productions. The initial auditory productions are 609 selected as auditory goals, then motor commands are computed 610 with a sensorimotor model that represents very well those ini611 tialization samples. Later, as the agent explores the auditory 612 space and it moves toward farther regions from those of initial613 ization, the competence values might slightly decrease. This 614 is due to the incremental learning of probabilistic models and ${ }_{615}$ depends on the values assigned to the forgetting rates $\alpha_{\mathrm{SM}}$ and ${ }_{616} \alpha_{\mathrm{SS}}$. If these forgetting rates are close to zero, then the agent 617 is less prone to update its knowledge when new data are far 618 from the current knowledge. On the other hand, if the forget619 ting rates are high, then the agent will adapt its model to the 620 new data very fast but also it will forget faster its previous 621 knowledge since it is not reinforced.

622 We also argue that one of the reasons the propriocep623 tive agents perform better is the null competence produced 624 by nonphonatory vocalizations. The nonproprioceptive agent 625 produces much more undesired contacts (four times more

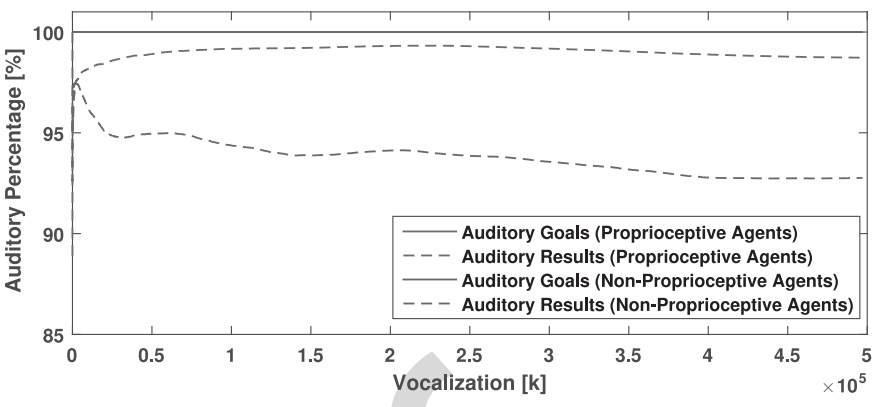

Fig. 6. Mean percentage of vocalizations producing phonatory result along all the simulations per each group, proprioceptive and nonproprioceptvie agents. The percentage of phonatory auditory goal is also shown per each group. Note: red and blue solid lines are overlapped.

undesired contacts on average) and, therefore, has more non- 626 phonatory vocalizations as corroborated in Fig. 6. Fig. 6 was 627 obtained using the same procedure than Fig. 4. It shows the 628 mean percentage of phonatory goals and actual phonatory 629 vocalizations considering all the simulations per each group 630 of artificial agents. In general, all the auditory goals through 631 the simulations are phonatory. The reason is that nonphonatory 632 goals become uninteresting very early in the artificial agent's 633 life as they are very easy to be produced. Thus, all those 634 nonphonatory vocalizations which produce null competence 635 impact negatively the average competence. We also might 636 think about all those nonphonatory vocalizations as a waste 637 of energy during the exploration. Knowing which regions of 638 the motor space are leading to collisions might be a relevant 639 knowledge for the agent. However, as the nonproprioceptive 640 agents keep exploring conflicting regions, the proprioceptive 641 agents avoid exploitation of these regions due to their ability to 642 predict somatosensory results from a given motor command. 643

Additionally, discussion about the tradeoff between explo- 644 ration and exploitation can be detailed. We argue that pro- 645 prioceptive agents show a better performance with respect to 646 exploitation, as agents avoid exploring uninteresting regions 647 with high number of contacts. In other words, propriocep- 648 tion, and in general constraint awareness, contributes to the 649 agent finding regularities faster and then fosters specialization 650 in regions of the auditory space, where the agent competence 651 to reach self-generated goals is higher. It is worth mentioning 652 that we are aware that it is also important to include the social 653 factor in the learning development of the artificial agent, in 654 order to better understand the role of proprioception in social 655 learning. In social learning, exploration is not just driven by 656 the progress in competence and discovery of constraints, but 657 also by the relevance of auditory goals for socialization pur- 658 poses. These studies leading to more exploring behaviors is 659 left for future work.

660

Furthermore, Fig. 5(b) shows the mean number of goals 661 rejected by the proprioceptive mechanism, represented in 662 lines 5-12 of Algorithm 1. In this plot, we prefilter the results 663 for proprioceptive agents considering a 5000 samples mov- 664 ing average for visualization purposes. It can be observed in 665 Fig. 5(b) that in general the proprioceptive mechanism is more 666 active at the beginning of the simulations presumably due to 667 the quantity of contacts along the initial set of vocalizations. 668 
669 Thus, we might deduce that proprioception prevents the agent 670 from further exploration in regions that are producing unde671 sired contacts especially in the early stages. In the next, we 672 introduce some figures in order to show the implications over 673 the shape of the explored auditory region when proprioception 674 is considered.

\section{B. On Explored Regions}

676 Regarding the volume of the explored region, Table I 677 indicates that the ratio of average volume of convex hulls 678 described by the explored regions in the frequency space is $6790.51 / 0.48$ between the nonproprioceptive and proprioceptive 680 agents, whereas the ratio of the mean competence is $0.53 / 0.71$. 681 In other words, whereas proprioceptive agents explore a $5.88 \%$ 682 tighter region than the nonproprioceptive, their performance 683 is $25.35 \%$ better than the later ones. On the other hand, 684 Table II considers only the vocalizations without undesired 685 contacts. A shrinkage of the convex hulls is observed, the 686 ratio of average volumes is, in this case, $0.40 / 0.39$ while 687 the mean competence ratio is $0.67 / 0.75$. From these numbers 688 we observe that, in general, the competence to vocalizations 689 without undesired contacts is higher for both kinds of agents. 690 However, regarding competence the proprioceptive agents still ${ }_{691}$ perform $11.94 \%$ better than the nonproprioceptive agents.

692 Based on Table I, we selected three different initial sets ${ }_{693}$ given their simulation results in order to produce Figs. 7-9. 694 First, we select initial set 1, as it performs better in terms 695 of competence when proprioception is considered. Second, to 696 contrast with initial set 1 we select initial set 5 , as its proprio697 ceptive agent performs the worst with regards to the percentage 698 of undesired contacts. Finally, we select initial set 9, as its 699 proprioceptive agent produced the largest convex hull volume. 700 Figs. 7-9 show some projections of vocalizations distribu701 tion maps of the auditory productions generated along the 702 simulation. Points in the plots are colored according to the 703 percentage of undesired contacts produced in its neighbor704 hood. Specifically, three projections are shown for each of 705 the selected sets of initial vocalizations. Projection $F_{1,1} F_{2,1}$ 706 represents the auditory fingerprint of the vocalizations in the 707 first perceptual window. Projections $F_{1,2} F_{2,2}$ is similar to the 708 first projection but for the second perceptual window. Finally, 709 projection $I_{1} I_{2}$ represents the value of the intonation parameter 710 in the first perceptual window against the same parameter in 711 the second perceptual window.

712 Distributions in Figs. 7-9 indicate that the intonation param713 eter projection $I_{1} I_{2}$ is the most influenced sensory-output due 714 to the proprioceptive feedback. Recall that $I_{1}$ and $I_{2}$ depend 715 on the average audibility of the vocalization which is null in 716 two cases: 1) when the voicing parameters are lower than zero 717 or 2) when the area function of the vocal tract is nonpositive 718 elsewhere. Therefore, keeping in mind the latter case, those 719 vocalizations producing an intonation parameter (either $I_{1}$ or $720 I_{2}$ ) lower than one indicate that a contact has likely occurred. ${ }_{721}$ If a contact occurs, there are two possible results, the average 722 of the minimum value of the area function might be nega723 tive or not. If it is negative, then the contact is classified as an 724 undesired contact and the proprioceptive signal takes the value
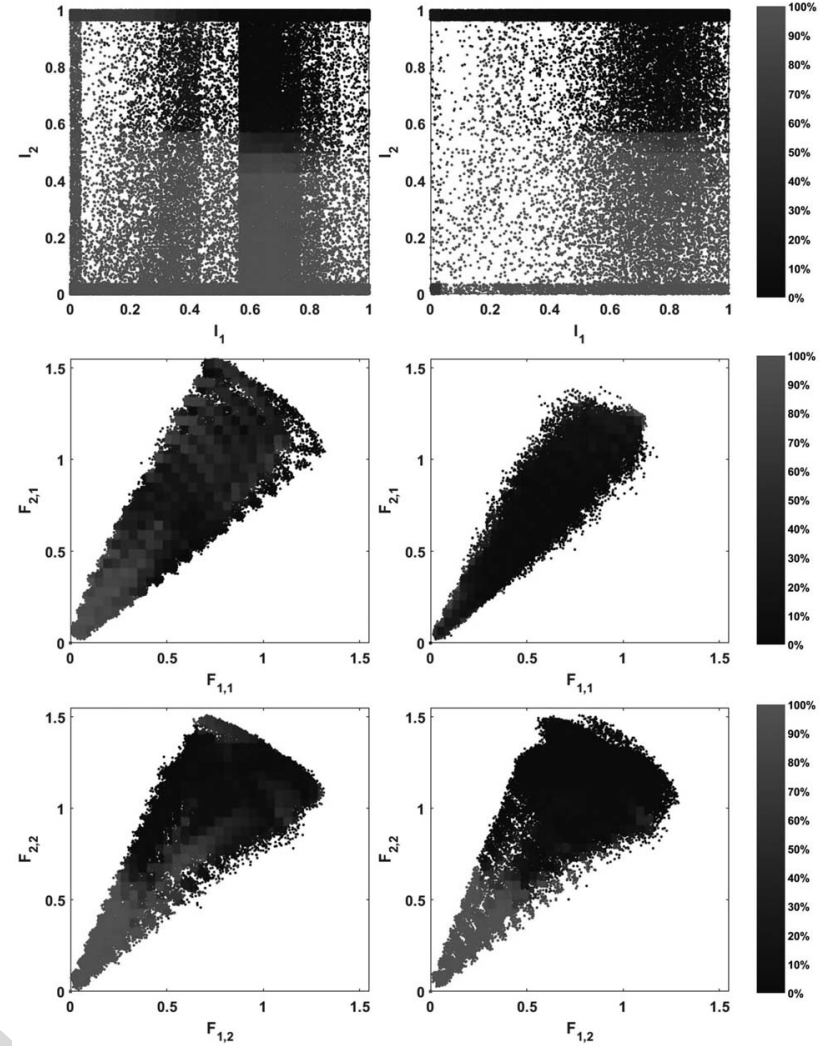

Fig. 7. Projections of vocalizations distribution along simulations using initial set 1 with Algorithm 1. Results for nonproprioceptive agent (left) and proprioceptive agent (right). Points are colored according to the total percentage of undesired contacts in their neighborhood.

one. Thus, having both values lower than one at the same time 725 is even more likely to produce undesired contacts. That is the 726 reason proprioceptive agents explore less intensively the mid- ${ }_{727}$ dle of the region in the intonation space. However, we argue 728 that in spite of the low density of vocalizations in that region, 729 proprioceptive agents succeed in finding more vocalizations 730 that produces nonconflicting articulatory configurations in that 731 region. For instance, looking at the projections in Fig. 9, the 732 proprioceptive agent almost covers all the intonation space ${ }_{733}$ with low density of contacts.

Moreover, comparing proprioceptive and nonproprioceptive 735 agents in Figs. 7-9, we observe that the area of the explored 736 regions varies slightly due to the proprioceptive mechanism. ${ }_{737}$ This fact is supported by Tables I and II. In general, in most ${ }_{738}$ of the cases using the proprioceptive feedback results in a ${ }_{739}$ slightly smaller explored region but this is not a conservative 740 fact. For instance, Table I indicates that the convex hull vol- 741 umes described in the auditory space by the experiments 2, 742 4 , and 9 were larger when proprioception was considered. In 743 general, besides a certain degree of randomness due to our 744 probabilistic approach, we argue that there are three main ele- 745 ments that determine the shape of the explored region: 1) the 746 initial set of vocalizations; 2) evolution of competence; and 747 3) proprioception.

748

Regarding the initial set, our criteria to choose random 749 vocalizations close to the neutral positions produces rich sets 750 of phonatory vocalizations, either with contacts or without. 751 

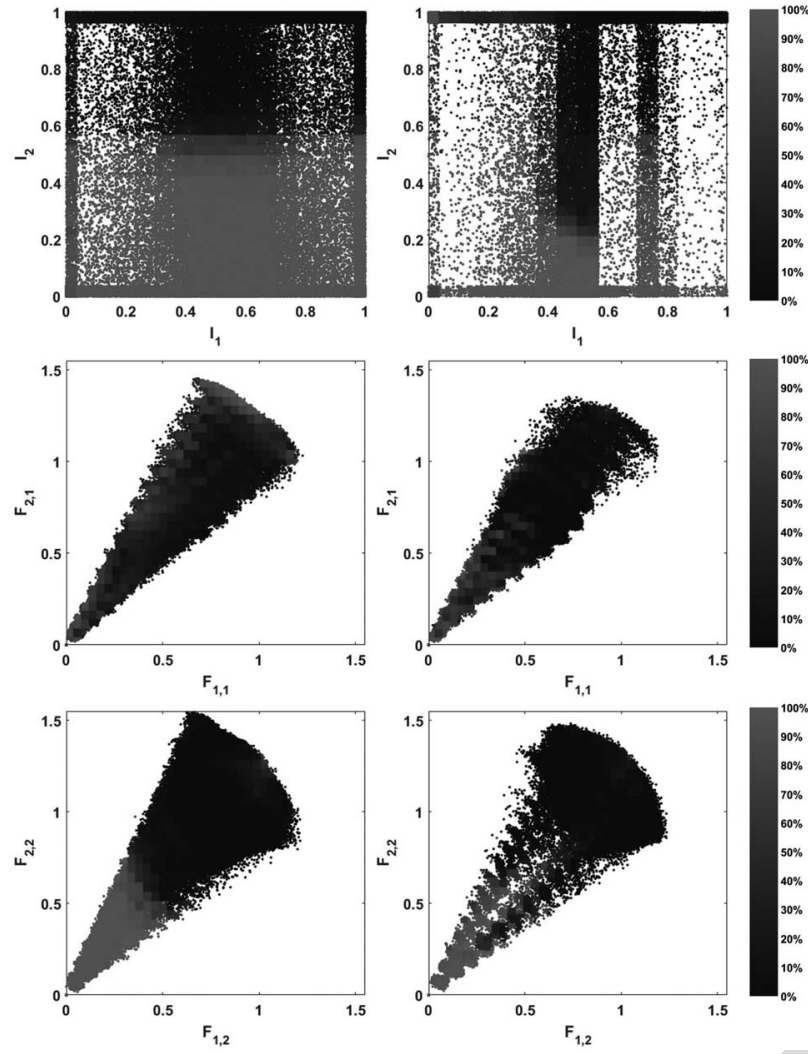

Fig. 8. Projections of vocalizations distribution along simulations using initial set 5 with Algorithm 1. Results for nonproprioceptive agent (left) and proprioceptive agent (right). Points are colored according to the total percentage of undesired contacts in their neighborhood.

752 As we are working with self-generated goals, the agent is 753 expected to be very good at the beginning at reaching goals 754 when auditory goals are close to the initialization region. In 755 the case of nonproprioceptive agents, the only parameter that 756 drives the exploration is the evolution of competence, which 757 is why we observe plenty of areas with a huge amount of 758 undesired contacts in the plots of nonproprioceptive agents 759 with respect to the proprioceptive agents. Furthermore, we 760 observe that proprioception might lead toward two different 761 situations: 1) an unexplored auditory region or 2) explored 762 region but with nonconflicting articulatory configurations. For 763 instance, in Fig. 7 , specifically in the projection $F_{1,1} F_{2,1}$, we ${ }_{764}$ observe that in general the nonproprioceptive agents produce 765 a lot of undesired contacts over almost the whole explored 766 region. On the contrary, the proprioceptive agent explores a 767 smaller region over the same projection, however it achieves 768 a considerably lower density of undesired contacts; results also 769 supported by the convex hull volume displayed in Table I. In 770 addition, projection $F_{1,2} F_{2,2}$ shows similar explored regions 771 for both agents. Indeed, the proprioceptive agent explored a 772 wider region in that projection and was capable of finding non773 conflicting vocalizations for some of the regions, where the 774 nonproprioceptive agent produces a lot of undesired contacts. 775 We observe, in general, for all the agents in Figs. 7-9, that 776 producing auditory results for the projection $F_{1,2} F_{2,2}$ close to 777 the origin is hard without producing contacts.
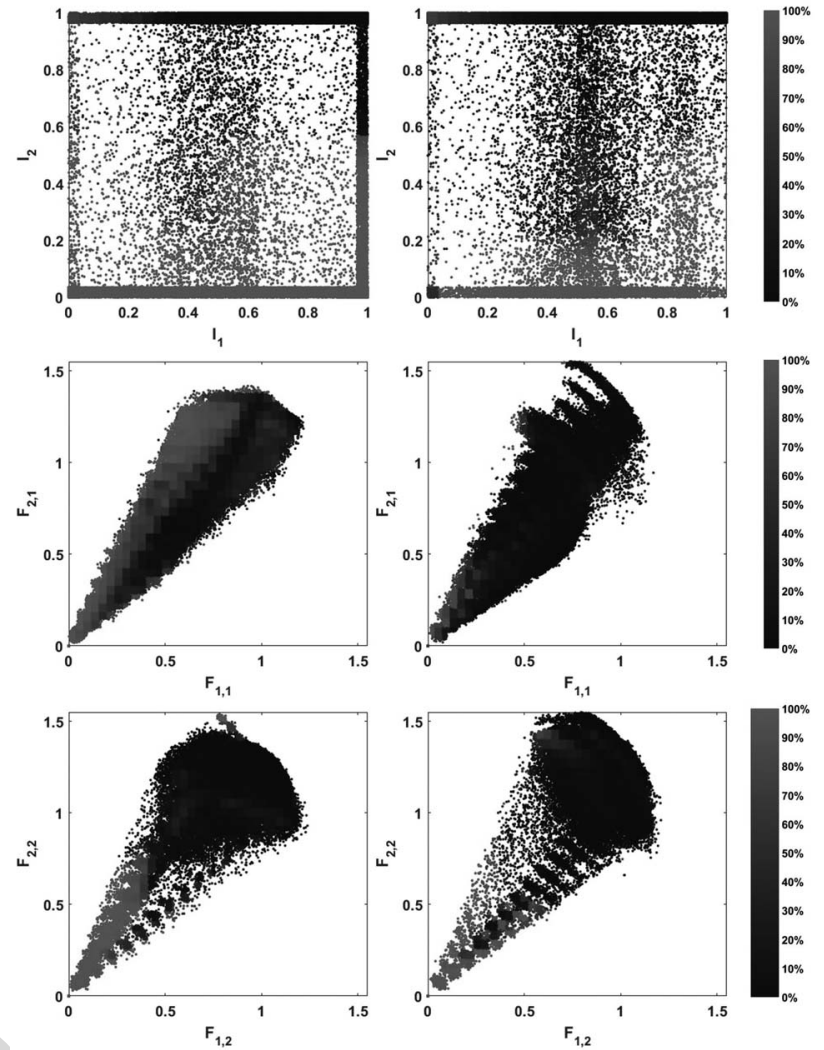

Fig. 9. Projections of vocalizations distribution along simulations using initial set 9 with Algorithm 1. Results for nonproprioceptive agent (left) and proprioceptive agent (right). Points are colored according to the total percentage of undesired contacts in their neighborhood.

In Fig. 8, corresponds to the agent with the worst results 778 using proprioception regarding the number of undesired con- 779 tacts, the projection $F_{1,1} F_{2,1}$ indicates that the proprioceptive 780 agent has explored a smaller region than the nonproprioceptive 781 agent. However, if we observe the boundaries of the explored 782 region with proprioception, they coincide with regions where 783 the nonproprioceptive agent produces a high amount of unde- 784 sired contacts. Thus, the proprioceptive mechanism does not 785 allow the proprioceptive agent to exploit those regions. In spite 786 of less exploration, we observe that in the explored region 787 where both agents intersect, the proprioceptive agent produces 788 less undesired contacts. Looking at Table II, the results for 789 the agent corresponding to Fig. 8 (experiment 5), we observe 790 that the explored regions with and without proprioception are 791 described by convex hulls with similar volume. This suggests 792 that the conflicting region explored by the nonpropriocep- 793 tive agent prevents the agent from exploiting regions without 794 undesired contacts. Thus, the agent achieve lower competence 795 values over the latter regions. On the other hand, the propri- 796 oceptive agent avoids conflicting regions, in consequence it 797 produces $15 \%$ less contacts and achieve a higher competence 798 average.

799

In addition, looking at the projection $F_{1,1} F_{2,1}$ Fig. 9, we 800 observe that the proprioceptive agent explores a larger region. 801 Moreover the density of contacts along the explored region is 802 significantly lower. This is also supported by the numerical 803 results in Tables I and II. On the other hand, in the projection 804 


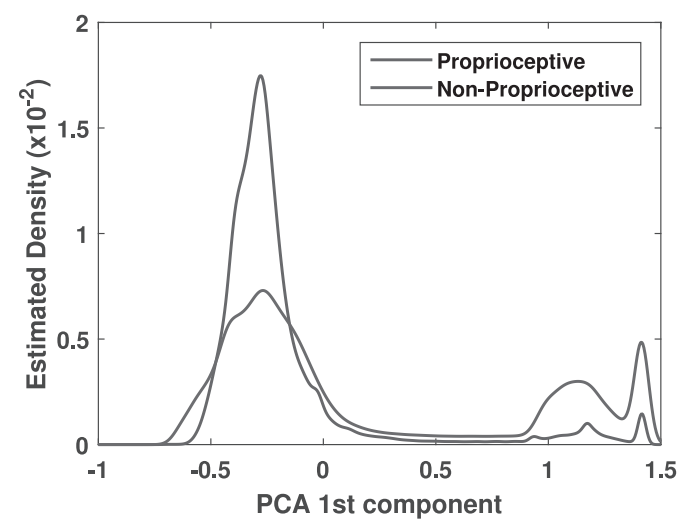

Fig. 10. Density distribution computed using Gaussian-kernels over all the data obtained along the simulations considering the first principal component with a variance contribution ratio of 0.68 .

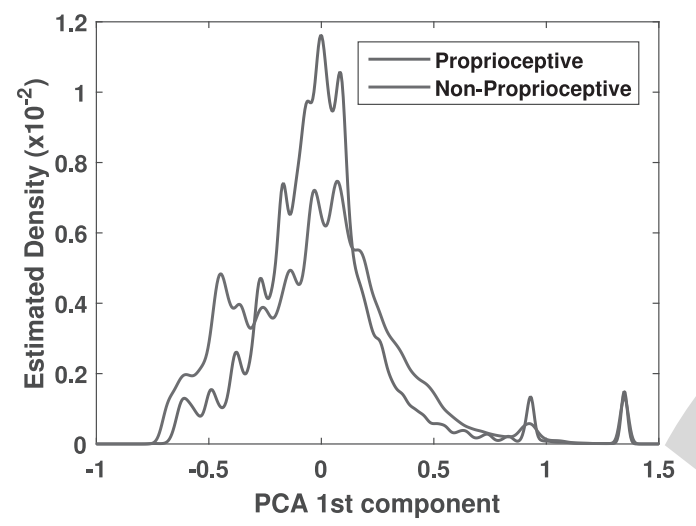

Fig. 11. Density distribution computed using Gaussian-kernels over the data, excluding vocalizations with undesired contacts, obtained along the simulations considering the first principal component with a variance contribution ratio of 0.61 .

$805 F_{1,2} F_{2,2}$ the exploration close to the origin of that projection 806 is less intensive in the proprioceptive agent, which reinforces 807 the previous observation of the difficulties to produce audi808 tory results in that region without contacts, similar results are 809 observed in Fig. 8. Future work also must focus in the study 810 of what is happening in that region and how relevant it is to 811 language, as well as modify the system accordingly.

${ }_{812}$ Finally, in order to observe the differences between the 813 vocalization distributions obtained using the different explo814 ration algorithms, we perform a sample density analysis over 815 the formant frequency dimensions. In order to make the results 816 easier to visualize we perform a principal component analysis 817 (PCA) procedure. We consider analyzing the data twice, first, 818 considering all the data collected along the exploration and 819 second, without considering the vocalizations with undesired 820 contacts. The PCA is done considering the dimensions $F_{1,1}$, $821 F_{2,1}, F_{1,2}$, and $F_{2,2}$, the data of all the 18 simulations are con822 catenated and used to perform the PCA. The PCA considering ${ }_{823}$ all the samples is performed and the first component is kept, 824 which contributes to the variance with a ratio of 0.61 . A sec825 ond PCA is performed considering only the non conflicting 826 vocalizations, again only the first component is kept, since it 827 contributes to the variance with a ratio of 0.68 . Once PCA transforms 4-D data into 1-D data, kernel-distribution estima- 828 tion is performed using Gaussian-kernels according to [31] for 829 the proprioceptive and nonproprioceptive cases.

830

In Figs. 10 and 11, we can observe the density distributions 831 obtained separately with all the proprioceptive and the nonpro- 832 prioceptive agents. First in Fig. 10, the distribution considering 833 all the data obtained from all the experiments is shown. In gen- 834 eral, it is observed that the agents explored similar regions, 835 but with different intensity. In Fig. 11, we observe the distri- 836 bution of the first component obtained from the PCA when ${ }_{837}$ only nonconflicting vocalizations are considered. In the latter 838 case it is observed that regarding the regions which are of 839 interest, in other words the regions where physical constraints 840 are not violated, both kinds of agents explore with a simi- 841 lar density shape, which means that even though both agents 842 explore similar interesting regions, the proprioceptive agents ${ }_{843}$ achieve in general higher competence.

\section{CONCLUSION}

845

An application of active learning techniques applied to the 846 study of vocal exploration considering motor constraints has 847 been introduced. It has been presented as an intrinsically moti- ${ }_{848}$ vated sensorimotor self-exploration architecture with motor 849 constraints self-awareness. Constraints awareness is achieved 850 by providing a proprioceptive mechanism which endows an 851 artificial agent with the capacity to autonomously generate a 852 somatosensory model. This model is then used to predict the ${ }_{853}$ consequences of a motor action and to avoid its execution if 854 it is expected to generate an undesired proprioceptive result. 855

The proprioceptive mechanism improved the quality of 856 learning according to a competence function. However, we ${ }_{857}$ observe a tradeoff between exploration and exploitation, 858 predominantly nonproprioceptive agents achieve greater explo- 859 ration in the auditory space. In contrast, we observe a more 860 intensive exploitation in interesting regions driving to the 861 higher competence values achieved by proprioceptive agents. 862 In general, vocal-auditory spaces are high dimensional redun- 863 dant spaces, thus an auditory output may be produced by 864 different articulatory configurations. Some of these articula- 865 tory configurations may lead to undesired contacts. Hence, we 866 argue that sensorimotor redundancy is reduced when proprio- 867 ception is included in the system allowing the agent to focus on 868 exploitation of nonconflicting vocalizations. In consequence, 869 the sensorimotor model generated through the exploration does 870 not include conflicting regions, where constraint violations are 871 likely to happen. For that reason, sensorimotor models achieve 872 better fitting to the regions of interest where constraints are 873 met. In this way, we showed how sensorimotor exploration, 874 and in general sensorimotor knowledge, can be shaped by 875 constraints.

Regarding the advance toward vocal exploration, we have 877 showed the suitability of the presented architecture to learn 878 vocal spaces in interesting and less redundant regions as chil- 879 dren might do. However, in order to continue our research 880 on early vocal development, we must study in greater depth 881 the first period of vocalization development. A deeper anal- ${ }_{882}$ ysis of the learning processes underlying the nonauditory 883 
884 development related to mastication, deglutition, and crying 885 from the cognitive and developmental perspectives should be 886 completed in order to generate more complex somatosensory 887 architectures. Finally, the next step of this paper should be 888 directed toward the self-structuring of vocalization and social 889 learning.

\section{REFERENCES}

[1] P. K. Kuhl, "Early language acquisition: Cracking the speech code," Nat. Rev. Neurosci., vol. 5, no. 11, pp. 831-843, 2004.

2] M. Asada et al., "Cognitive developmental robotics: A survey," IEEE Trans. Auton. Mental Develop., vol. 1, no. 1, pp. 12-34, May 2009.

[3] R. Pfeifer, M. Lungarella, and F. Iida, "Self-organization, embodiment, and biologically inspired robotics," Science, vol. 318, no. 5853, pp. 1088-1093, 2007.

[4] R. Pfeifer and C. Scheier, Understanding Intelligence. Cambridge, U.K.: MIT Press, 1999.

[5] D. A. Cohn, Z. Ghahramani, and M. I. Jordan, "Active learning with statistical models," J. Artif. Intell. Res., vol. 4, no. 1, pp. 129-145, 1996.

[6] S. Thrun, "Exploration in active learning," in Handbook of Brain Science and Neural Networks, 1995, pp. 381-384.

[7] C. Moulin-Frier and P.-Y. Oudeyer, "Exploration strategies in developmental robotics: A unified probabilistic framework," in Proc. Int. Conf. Develop. Learn. (ICDL/Epirob), Osaka, Japan, 2013, pp. 1-6.

[8] J. M. Acevedo-Valle, C. Angulo, N. Agell, and C. Moulin-Frier, "Proprioceptive feedback and intrinsic motivations in early-vocal development," in Proc. 18th Int. Conf. Catalan Assoc. Artif. Intell., 2015, pp. $9-18$.

[9] C. Moulin-Frier, S. M. Nguyen, and P.-Y. Oudeyer, "Self-organization of early vocal development in infants and machines: The role of intrinsic motivation," Front. Psychol., vol. 4, pp. 1006-1025, Jan. 2014 doi: 10.3389/fpsyg.2013.01006.

10] F. H. Guenther, S. S. Ghosh, and J. A. Tourville, "Neural modeling and imaging of the cortical interactions underlying syllable production," Brain Lang., vol. 96, no. 3, pp. 280-301, 2006.

[11] A. S. Warlaumont, G. Westermann, E. H. Buder, and D. K. Oller, "Prespeech motor learning in a neural network using reinforcement," Neural Netw., vol. 38, pp. 64-75, Feb. 2013.

12] B. J. Kröger, J. Kannampuzha, and C. Neuschaefer-Rube, "Towards a neurocomputational model of speech production and perception," Speech Commun., vol. 51, no. 9, pp. 793-809, 2009.

[13] I. S. Howard and P. Messum, "Modeling the development of pronunciation in infant speech acquisition," Motor Control, vol. 15, no. 1, pp. 85-117, 2011.

[14] M. Rolf, J. J. Steil, and M. Gienger, "Goal babbling permits direct learning of inverse kinematics," IEEE Trans. Auton. Mental Develop., vol. 2, no. 3, pp. 216-229, Sep. 2010.

15] A. Baranes and P.-Y. Oudeyer, "Active learning of inverse models with intrinsically motivated goal exploration in robots," Robot. Auton. Syst., vol. 61, no. 1, pp. 49-73, 2013.

16] C. Moulin-Frier and P.-Y. Oudeyer, "Learning how to reach various goals by autonomous interaction with the environment: Unification and comparison of exploration strategies," in Proc. 1st Multidiscipl. Conf. Reinforcement Learn. Decis. Making (RLDM), Princeton, NJ, USA, Oct. 2014, Art. no. hal-00922537. [Online]. Available: https://hal.inria.fr/hal-00922537/document
[17] A. Ribes, J. Cerquides, Y. Demiris, and R. Lopez de Mántaras, "Active 939 learning of object and body models with time constraints on a humanoid 940 robot," IEEE Trans. Cogn. Develop. Syst., vol. 8, no. 1, pp. 26-41, 941 Mar. 2016, doi: 10.1109/TAMD.2015.2441375.

[18] J. Perkell et al., "The sensorimotor control of speech production," 943 in Proc. 1st Int. Symp. Meas. Anal. Model. Human Functions, 2001, 944 pp. 359-365.

[19] D. K. Oller and R. E. Eilers, "The role of audition in infant babbling," 946 Child Develop., vol. 59, no. 2, pp. 441-449, 1988.

[20] K. Ejiri, "Relationship between rhythmic behavior and canonical 948 babbling in infant vocal development," Phonetica, vol. 55, no. 4, 949 pp. 226-237, 1998.

[21] P.-Y. Oudeyer, F. Kaplan, and V. V. Hafner, "Intrinsic motivation systems 95 for autonomous mental development," IEEE Trans. Evol. Comput., 952 vol. 11, no. 2, pp. 265-286, Apr. 2007. seeking, curiosity, and attention: Computational and neural mecha- 955 nisms," Trends Cogn. Sci., vol. 17, no. 11, pp. 585-593, 2013.

[23] B. Galantucci, C. A. Fowler, and M. T. Turvey, "The motor theory of 957 speech perception reviewed," Psychonomic Bull. Rev., vol. 13, no. 3, 958 pp. 361-377, 2006.

[24] J.-L. Schwartz, A. Basirat, L. Ménard, and M. Sato, "The perception- 960 for-action-control theory (PACT): A perceptuo-motor theory of speech 961 perception," J. Neurolinguist., vol. 25, no. 5, pp. 336-354, 2012.

[25] S. Tremblay, D. M. Shiller, and D. J. Ostry, "Somatosensory basis of 963 speech production," Nature, vol. 423, no. 6942, pp. 866-869, 2003.

[26] S. M. Nasir and D. J. Ostry, "Speech motor learning in profoundly deaf 965 adults," Nature Neurosci., vol. 11, no. 10, pp. 1217-1222, 2008.

[27] S. N. Iyer and D. K. Oller, "Prelinguistic vocal development in infants 967 with typical hearing and infants with severe-to-profound hearing loss," 968 Volta Rev., vol. 108, no. 2, pp. 115-138, 2008.

[28] T. Ito, M. Tiede, and D. J. Ostry, "Somatosensory function in speech 970 perception," Proc. Nat. Acad. Sci. USA, vol. 106, no. 4, pp. 1245-1248, 971 2009.

[29] C. Moulin-Frier and P.-Y. Oudeyer, "The role of intrinsic motivations in 973 learning sensorimotor vocal mappings: A developmental robotics study," 974 in Proc. Interspeech, Lyon, France, 2013, pp. 1268-1272.

[30] S. Calinon, Robot Programming by Demonstration. Lausanne, 976 Switzerland: EPFL Press, 2009.

[31] D. W. Scott, Multivariate Density Estimation: Theory, Practice, and 978 Visualization. Hoboken, NJ, USA: Wiley, 2015.

Juan Manuel Acevedo-Valle, photograph and biography not available at the 980 time of publication.

Cecilio Angulo, photograph and biography not available at the time of 982 publication.

Clement Moulin-Frier, photograph and biography not available at the time 984 of publication. 